\title{
R\&D ERL: Magnetic Measurements of the ERL Magnets
}

Animesh Jain, Superconducting Magnet Division

Brookhaven National Laboratory, Upton, NY 11973, USA

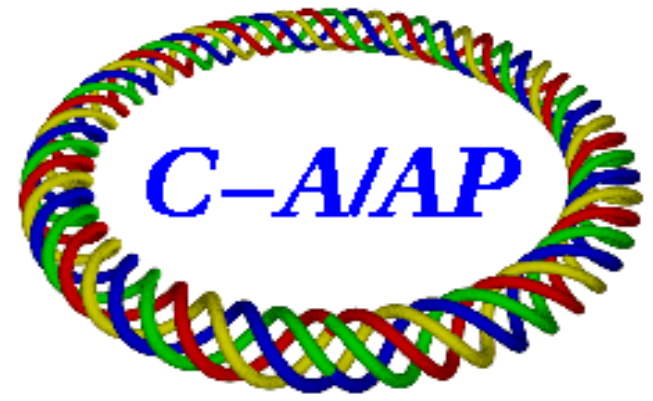

\section{Collider-Accelerator Department Brookhaven National Laboratory Upton, NY 11973}

Notice: This document has been authorized by employees of Brookhaven Science Associates, LLC under Contract No. DE-AC02-98CH10886 with the U.S. Department of Energy. The United States Government retains a nonexclusive, paid-up, irrevocable, world-wide license to publish or reproduce the published form of this document, or allow others to do so, for United States Government purposes. 


\section{R\&D ERL: Magnetic Measurements of the ERL Magnets Animesh Jain, Superconducting Magnet Division Brookhaven National Laboratory}

\section{Introduction:}

The magnet system of ERL consists of G5 solenoids, 6Q12 quadrupoles with $0.58 \mathrm{~T} / \mathrm{m}$ gradient, 3D60 dipoles with $0.4 \mathrm{~T}$ central field, 15 and 30 degree Z-bend injection line dipole/quadrupole combined function magnets, and extraction line magnets. More details about the magnets can be found in a report by G. Mahler [1]. Field quality in all the 6Q12 quadrupoles, 3D60 dipoles and the injection line magnets has been measured with either a rotating coil, or a Hall probe mapper. This report presents the results of these magnetic measurements.

\section{Mapping of the 3D60 Dipoles:}

The 3D60 dipoles have a gap of $30 \mathrm{~mm}$ (vertical) $\times 140 \mathrm{~mm}$ (horizontal) with a maximum central field of $0.4 \mathrm{~T}$. The good field region is $\pm 10 \mathrm{~mm}$ (vertical) $\times \pm 30 \mathrm{~mm}$ (horizontal). The magnets have an edge angle of 15 degrees, which causes the axially integrated dipole field to vary by approximately $\pm 6.8 \%$ over the $\pm 30 \mathrm{~mm}$ radial distance. The geometry of these magnets is not suitable for harmonic measurements using rotating coils. The vertical field in the midplane was therefore mapped using a custom built Hall probe field mapping system. The measurement system, and the results obtained, are summarized here.

\subsection{Hall Probe Mapping System:}

The system consists of a 3-axis translation stage and a Hall probe holder with four high precision Group3 MPT-141 probes. The stage can provide a total motion of $\pm 300 \mathrm{~mm}$ (axial) $\times \pm 125 \mathrm{~mm}$ (radial) $\times \pm 25 \mathrm{~mm}$ (vertical) with a resolution of $\pm 1 \mu \mathrm{m}$ and absolute position error of less than $\pm 25 \mu \mathrm{m}$. The four Hall probes are arranged such that they measure the field at different radial positions for the same axial position. A single axial scan of the probe thus provides the axial field profile at four different radial positions. The separation between two adjacent Hall probes is roughly $10 \mathrm{~mm}$. The exact separation between the probes is measured with an accuracy of a few microns by carrying out a scan in a quadrupole magnet at $\sim 4.8 \mathrm{~T} / \mathrm{m}$. The dipole magnet is mounted on a separate stand using two pins in the axial direction. The axis of the stand, as defined by the mounting pins, is aligned to the Hall probe translation system using precision dial gauges. The entire translation stage is mounted on a tilt table with yaw, pitch and roll adjustments, as shown in Fig. 1. This allows a relatively easy and accurate alignment of the Hall probe motion axes with the magnet axes.

\subsection{Absolute Calibration of the Hall Probes:}

One drawback of using a Hall probe array is the uncertainty in the relative calibration of the probes at a level of $0.01 \%$ or less. Although the Hall probes were previously tested and calibrated against NMR in a reference dipole field with good field uniformity, small changes in the calibration (within the manufacturer's specifications) were seen during the tests of the 3D60 dipoles. It was, therefore, decided to carry out a calibration of the four probes against NMR during measurement of each magnet. A separate excitation curve with a NMR probe 


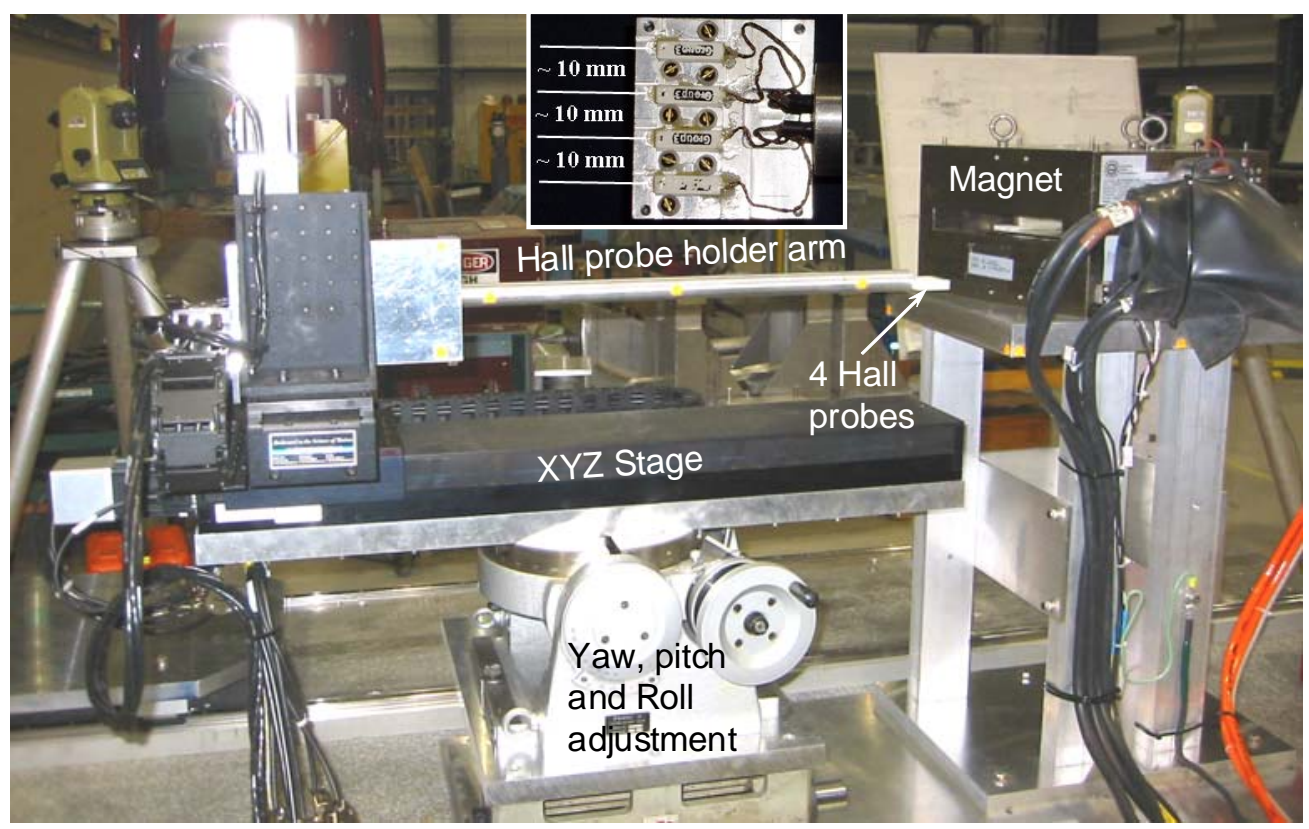

Fig. 1 Hall probe mapping system for the 3D60 dipoles. The inset shows the arrangement of the Hall probes.

placed next to the Hall probes was measured for this purpose, from which the absolute calibration of each probe was established. An example of calibration data is shown in Fig. 2.

\section{ERD004; NMR Check of Probes; 14-May-2008}

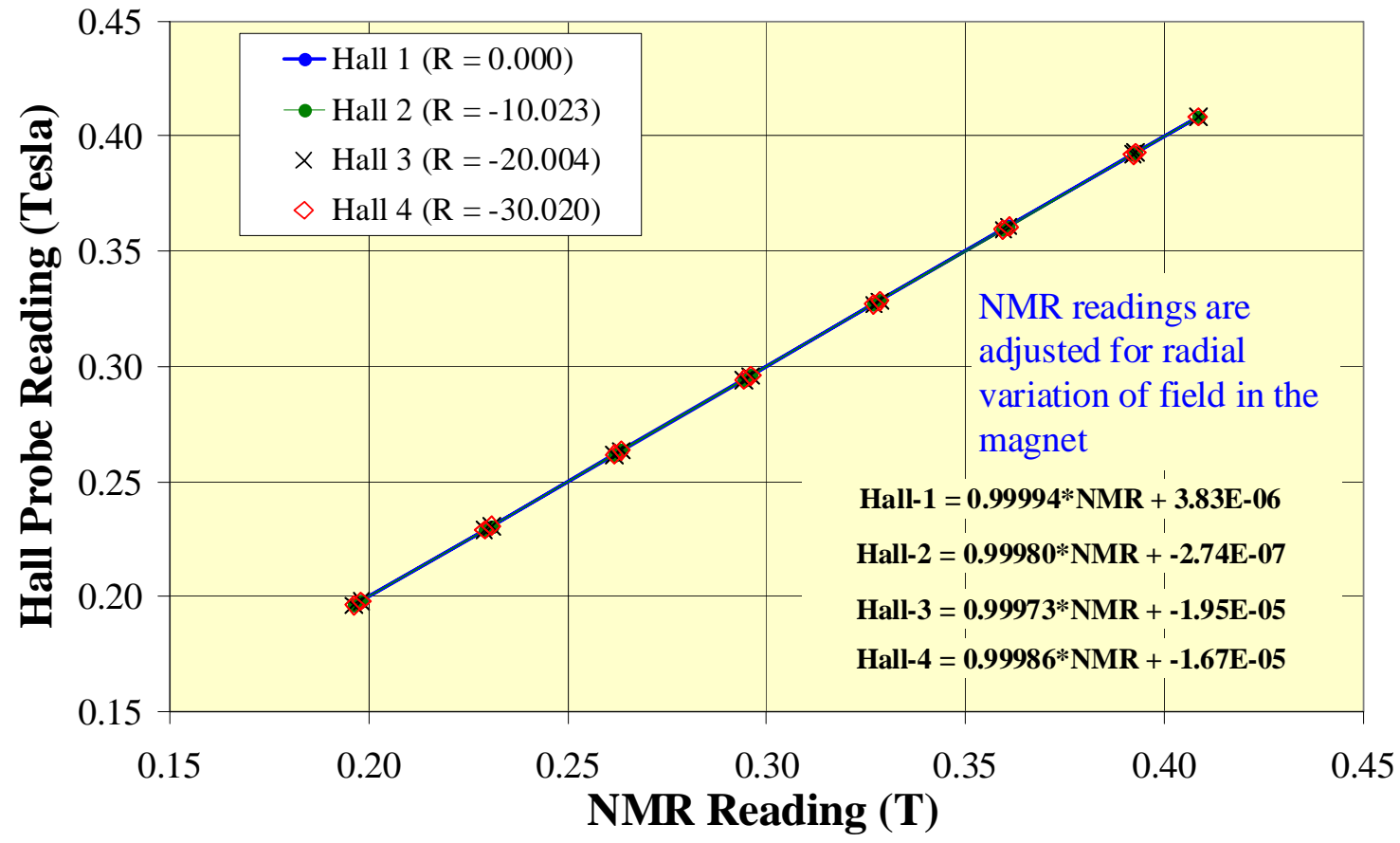

Fig. 2 Hall probe calibration against NMR in 3D60 dipole \#4. 


\subsection{Measurement of Excitation Curves at Magnet Center:}

The excitation curves of the magnet and hysteresis effects were measured with one of the Hall probes located at the magnet center. The magnet iron history was standardized by carrying out five powering cycles from $0.1 \mathrm{~A}$ to $250 \mathrm{~A}$ and then back to $0.1 \mathrm{~A}$ at $5 \mathrm{~A} / \mathrm{s}$. The current was then ramped in $5 \mathrm{~A}$ steps at $2.5 \mathrm{~A} / \mathrm{s}$. Two sets of Hall probe readings were taken at each current step, except at the maximum current of 250 A where 10 sets of readings were taken. The downward ramp of the excitation cycle was then measured in similar 5 A steps. The measurements in both the up and the down ramps of the excitation cycle were then repeated for another four cycles (a total of 5 cycles) to study any cycle to cycle variations in the field.

Fig. 3 shows an example of excitation curve data taken in 3D60 dipole \#5. The magnet reaches the maximum design field of $0.4 \mathrm{~T}$ at about $243 \mathrm{~A}$. In order to clearly look for the remnant field and any cycle to cycle differences, a term proportional to the current was subtracted out from the data. The so called "non-linear" part of the field is plotted in Fig. 4. The field in all the cycles is nearly identical, with typical differences between cycles being less than $20 \mu \mathrm{T}$, except for the first half of the up ramp in the very first cycle, where the difference from the second cycle may be up to $40 \mu \mathrm{T}$. The remnant field is approximately $1.5 \mathrm{mT}$. The results in all the 3D60 dipoles are similar to those shown in Figs. 3 and 4. The average transfer function in all dipoles is $1.641 \mathrm{mT} / \mathrm{A}$, with a standard deviation of $0.2 \%$. The transfer function shows some correlation with the measured gaps in the magnets. It should be noted that the excitation curve measurements were made in the center of the magnet, whereas the gaps were measured at the edges of the magnet. This could account for the fact that only a qualitative correlation with gap is found.

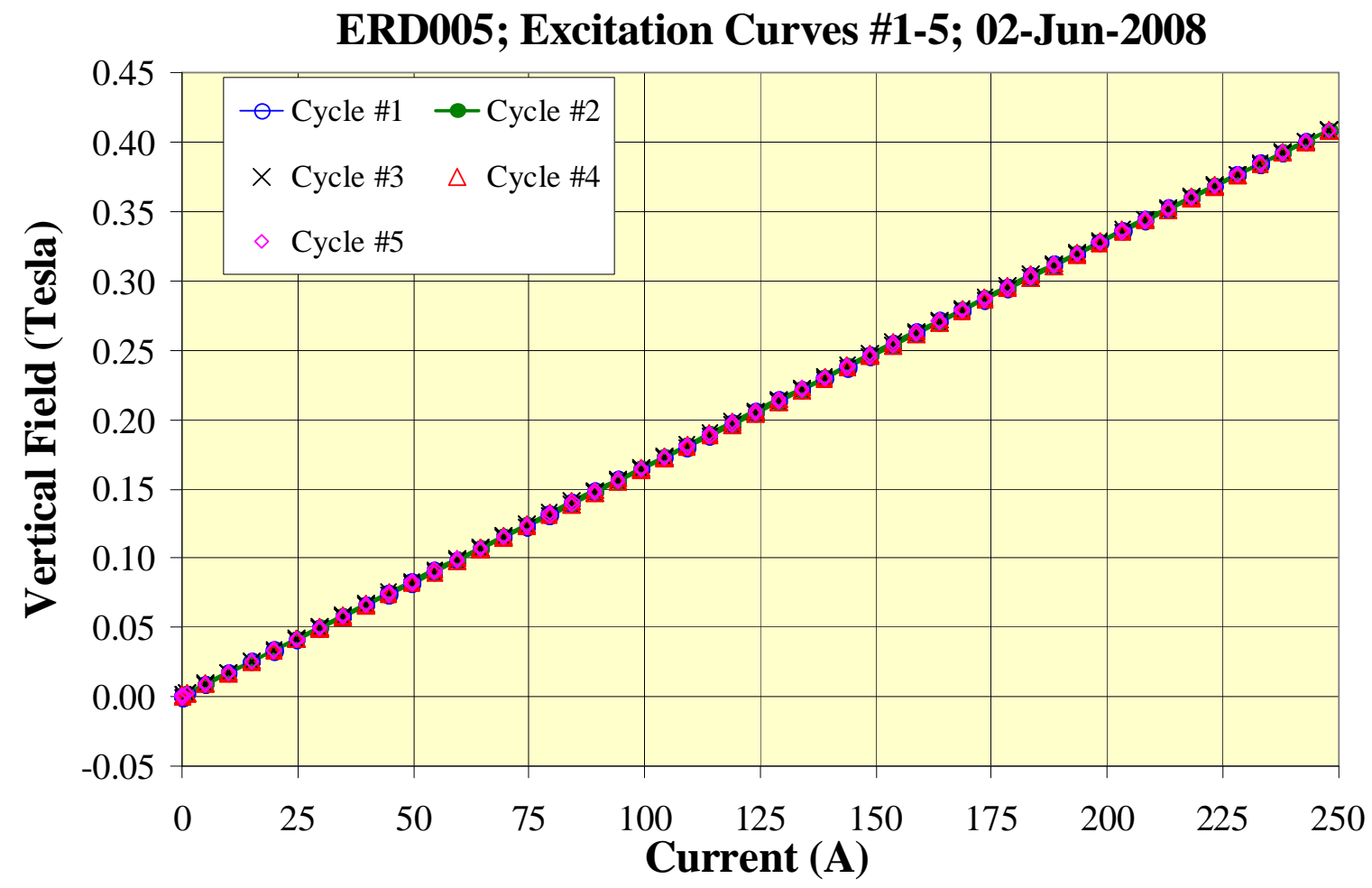

Fig. 3 Vertical field in the center of the magnet as a function of current for 5 successive excitation cycles in 3D60 dipole \#5. 


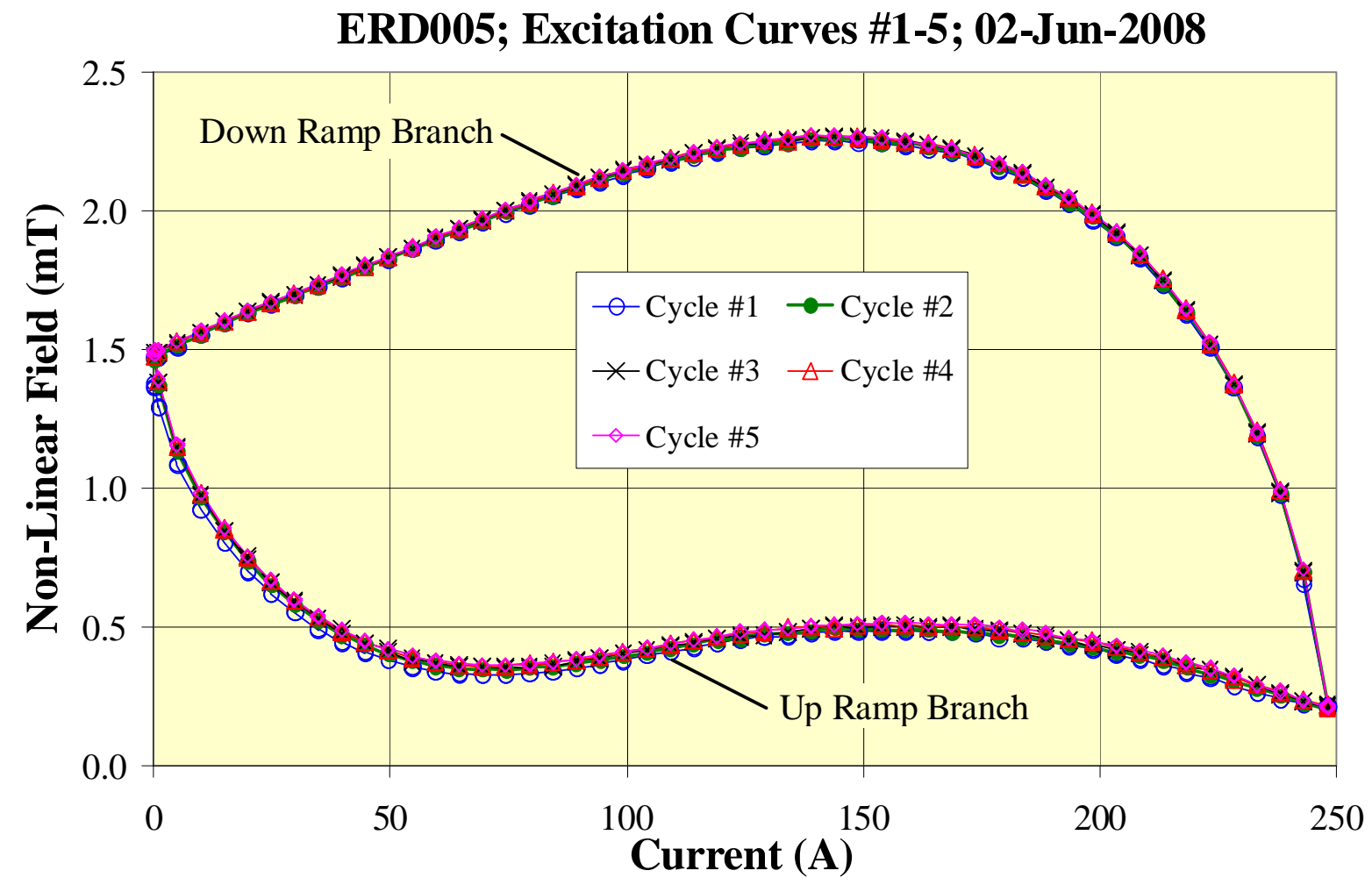

Fig. 4 Non-linear part of the vertical field in the center of the magnet as a function of current for 5 successive excitation cycles in 3D60 dipole \#5. No appreciable cycle to cycle difference is seen, except for the low current end of the up ramp branch in the very first cycle.

\subsection{Measurement of Radial Profile at Axial Center}

The local field homogeneity in the horizontal (radial) direction was measured at the axial center of the magnet at a fixed excitation of $205 \mathrm{~A}$ on the up ramp, which gives a central field of approximately $0.335 \mathrm{~T}$. Since the four Hall probes are also arranged along this scan direction, each of the Hall probes essentially measures the same field profile, except for the range of coverage provided. As the Hall probes have a separation of only $10 \mathrm{~mm}$ and the scan covers a range of $\pm 50 \mathrm{~mm}$, there is considerable overlap between the profiles measured by various probes. Even small differences in the probe calibrations show up very clearly in these results. With one of the Hall probes (the one closest to the NMR probe during calibration) treated as a reference, the calibrations of the remaining probes was further fine tuned such that the radial profiles measured by all the four Hall probes are identical. As an illustration, Figs. 5 and 6 show typical profiles in a 3D60 dipole before and after adjustments to the Hall probe calibrations. In general, the calibration factors obtained by fitting the profiles in this way were very close to those obtained by NMR. Due to excellent short term stability of the Group3 Hall probes, the field homogeneity, $\left(\Delta B_{y} / B_{y}\right)$, was measured with an uncertainty of well below $0.01 \%$ (approx. $0.002 \%$, or $20 \mathrm{ppm}$ ). Nearly all magnets showed the required field homogeneity of better than $\pm 0.01 \%$ over $\pm 30 \mathrm{~mm}$, with the largest deviation being less than $\pm 0.02 \%$ in one or two magnets. 
ERD006: Radial Scan at 0.335T; 23-May-2008

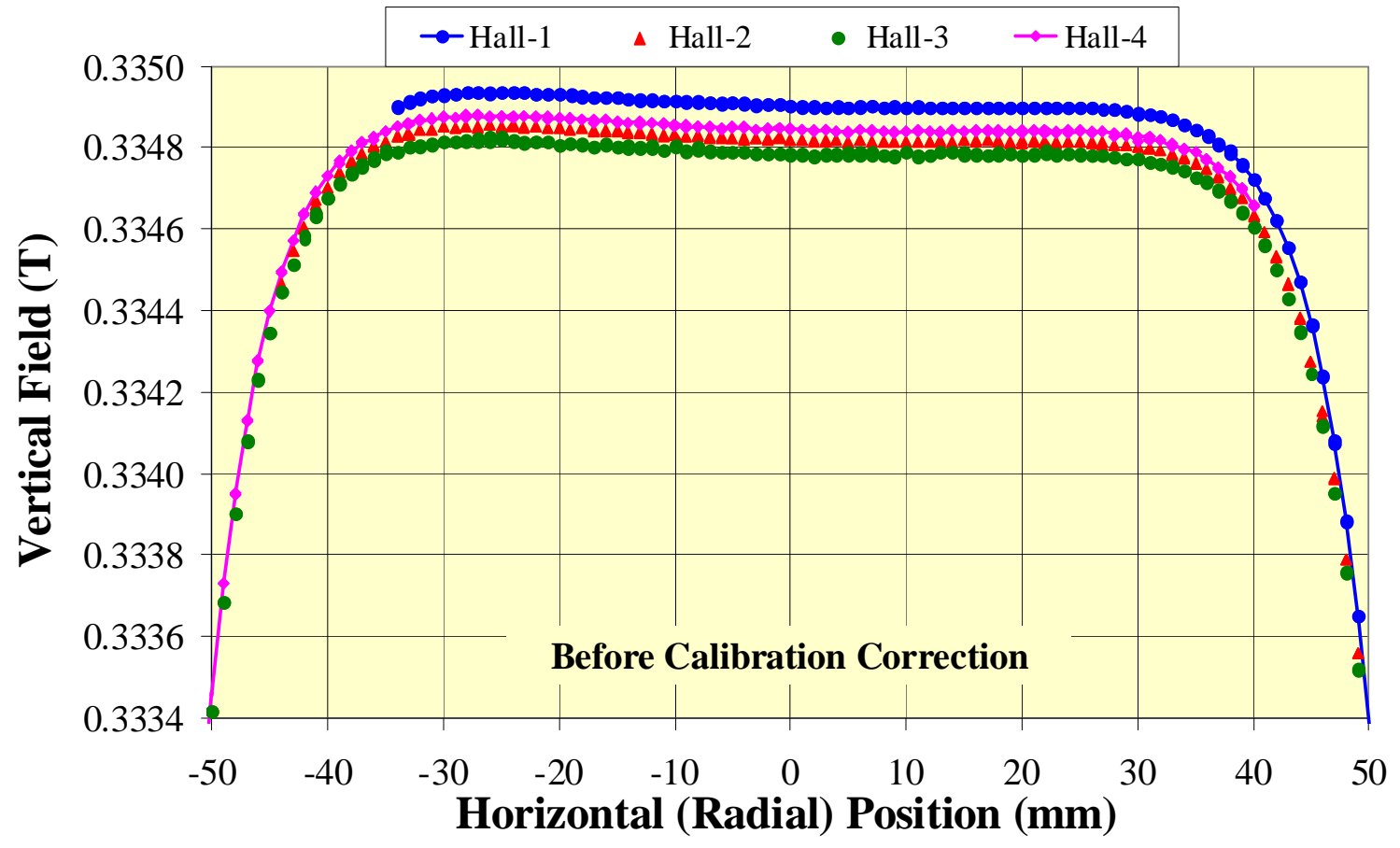

Fig. 5 Horizontal (radial) profiles of the vertical field measured by the four Hall probes in 3D60 dipole \#6 before calibration corrections are applied.

ERD006: Radial Scan at 0.335T; 23-May-2008

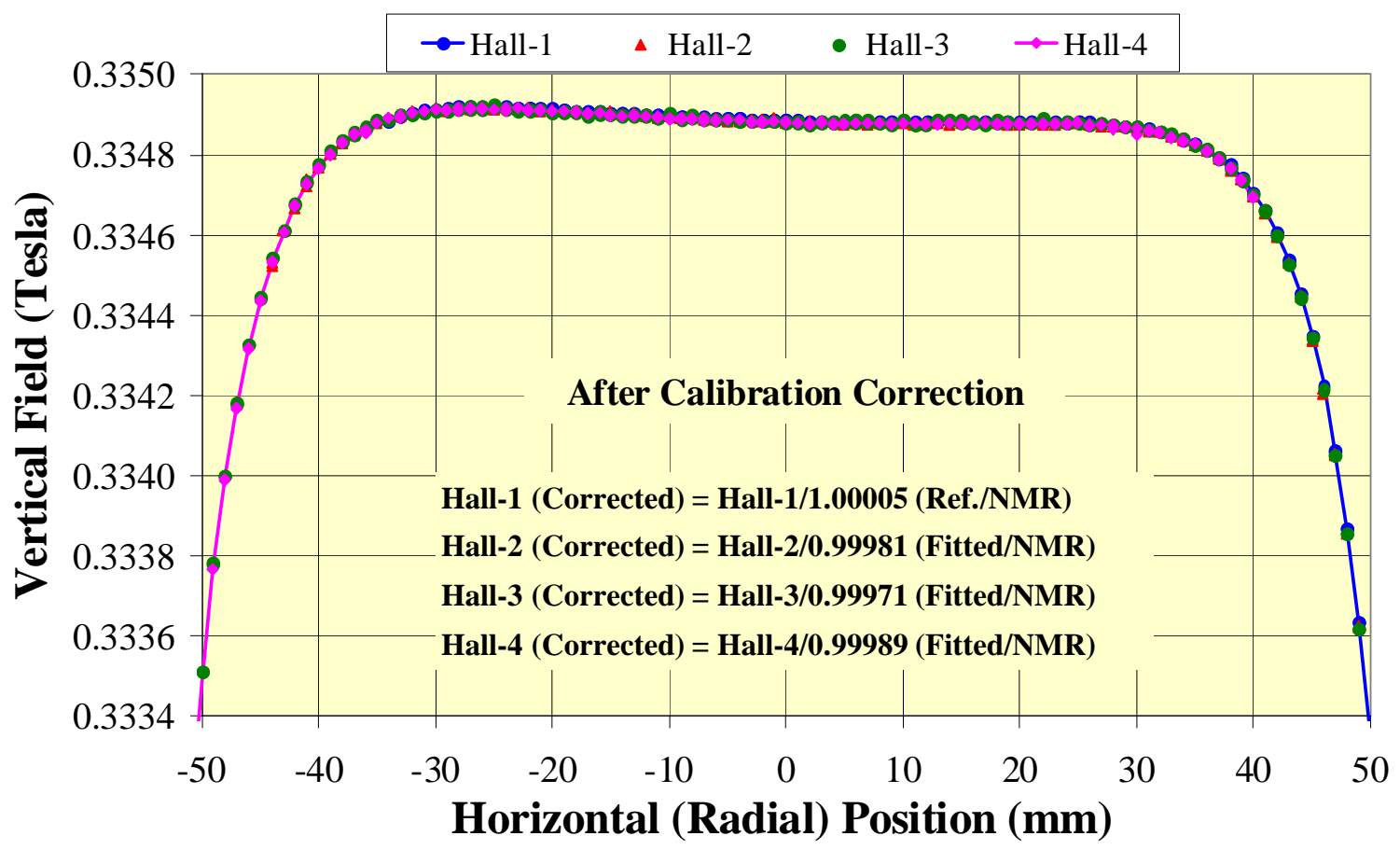

Fig. 6 Horizontal (radial) profiles of the vertical field measured by the four Hall probes in 3D60 dipole \#6 after calibration corrections are applied. 


\subsection{Measurement of Axial Profiles and Integral Field}

The axial profile of the vertical field component was measured in the region $\pm 250 \mathrm{~mm}$ in the axial direction. Since there are four Hall probes on the holder, each axial scan of the probe yields four axial profiles. A total of 12 such axial scans were sufficient to cover a region of $\pm 50 \mathrm{~mm}$ in the radial direction, with a few radial positions being measured by two different probes in two different scans. This feature allowed another built-in check of Hall probe calibration and probe positioning accuracy. The integrated fields measured by different Hall probes at a given radial positions agreed within $\pm 0.01 \%$, which includes the effect of Hall probe calibration errors, as well as any positioning uncertainty in the radial direction.

An example of the axial profile data is shown in Fig. 7. Only a small portion of the axial scan is plotted to clearly show the differences between the four radial positions measured in a single scan. This magnet shows a very slight taper $(0.02 \%)$ from one end to the other.

The integrated field computed by integrating the axial profile data is plotted as a function of radial position in Fig. 8. The data points measured by each Hall probe are shown by the respective symbols. It can be seen that a few of the points are measured by two different Hall probes in two different scans, with excellent consistency $( \pm 0.01 \%)$.

\section{ERD004_20080519_XScan05}

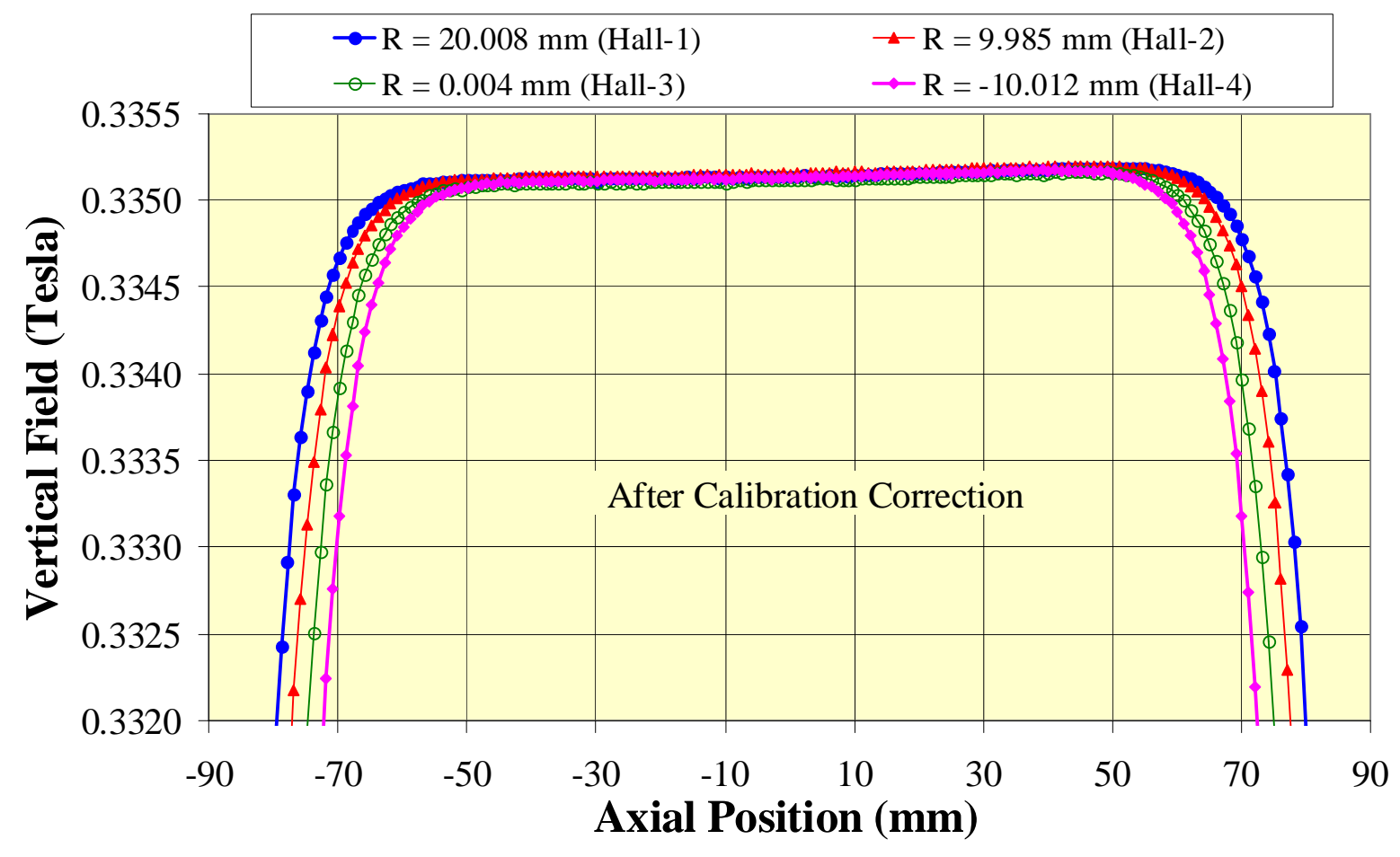

Fig. 7 Axial profiles of the vertical field measured by the four Hall probes in 3D60 dipole \#4 in one of the 12 axial scans that covers the region from $-10 \mathrm{~mm}$ to $+20 \mathrm{~mm}$ in $10 \mathrm{~mm}$ steps. 


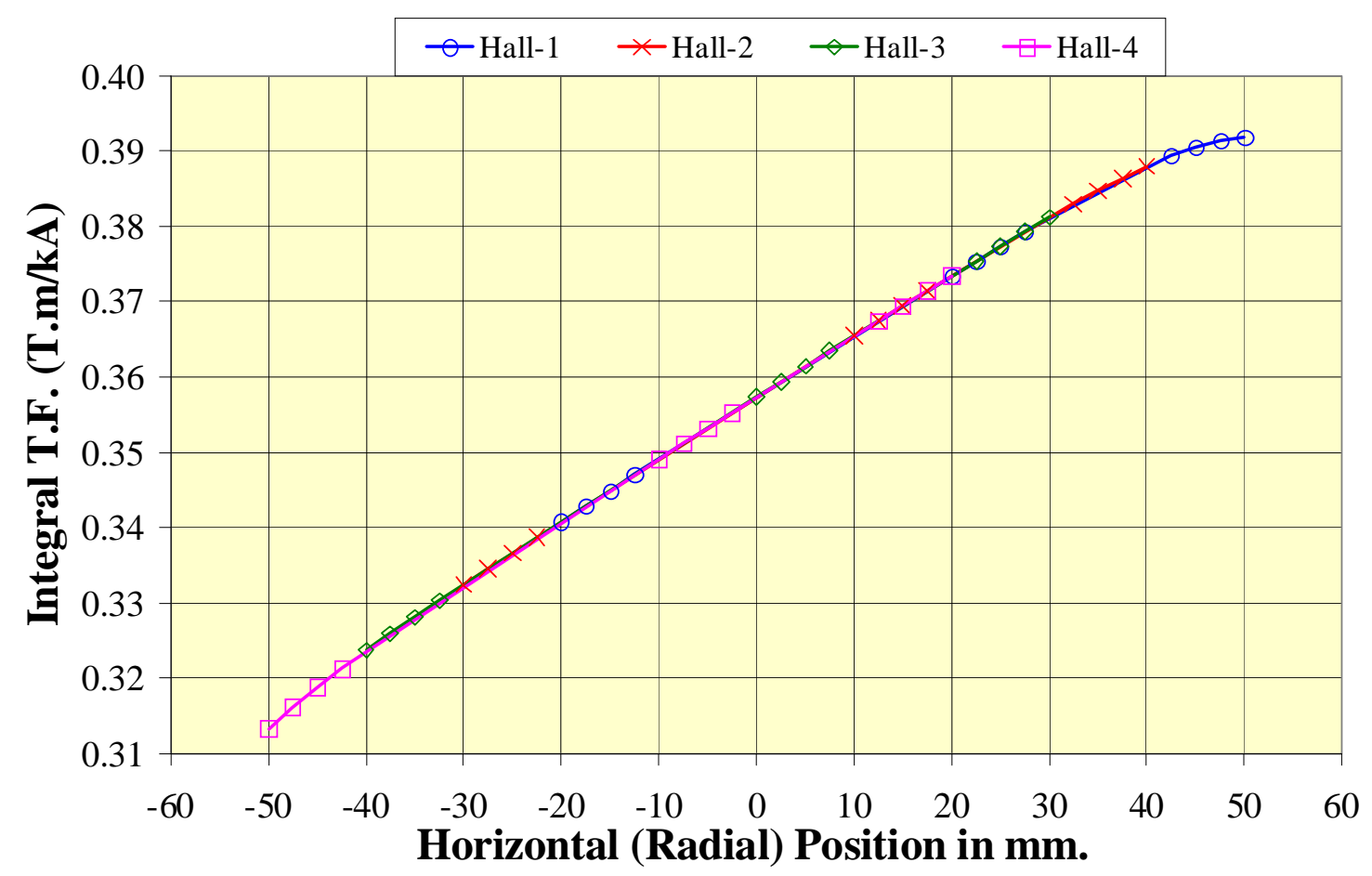

Fig. 8 Variation of the integral transfer function (integral field normalized to the current) as a function of radial position measured using 12 axial scans.

\subsection{Measurement of Excitation Curves of the Trim Coil:}

The 3D60 dipoles are also equipped with $2 \%$ trim coils for adjusting the field strength. Excitation curves for the trim windings were measured in the center of the magnet with the main coil set to $104 \mathrm{~A}$, producing $0.172 \mathrm{~T}$ field. Two complete up and down ramps were measured in the current range of $\pm 3 \mathrm{~A}$ in the trim coil in $0.1 \mathrm{~A}$ steps, after carrying out 5 cycles between $\pm 3 \mathrm{~A}$ at $0.1 \mathrm{~A} / \mathrm{s}$ to set the iron history. Fig. 9 shows an example of the excitation curve data taken in 3D60 dipole \#5. The amount of trim field produced at $3 \mathrm{~A}$ is approximately $8 \mathrm{mT}$, which is in agreement with the design value of $2 \%$ of the $0.4 \mathrm{~T}$ maximum main field. The hysteresis and reproducibility of the measurements can be seen more clearly in Fig. 10 which shows the non-linear component of the field after subtracting out a term proportional to the trim coil current. The cycle to cycle consistency is within $10 \mu \mathrm{T}$ in this case.

\section{Magnetic Measurements in the 6Q12 Quadrupoles:}

The 6Q12 quadrupoles have a $60 \mathrm{~mm}$ diameter aperture and a design gradient of $3 \mathrm{~T} / \mathrm{m}$ at $5.8 \mathrm{~A}$. The yoke length is $12.8 \mathrm{~cm}$ and the integrated gradient is $0.45 \mathrm{~T}$. The magnet also has trim coils on all the four poles which can be configured either as a vertical field (horizontal steering) or a horizontal field (vertical steering) dipole corrector. The integrated field quality in these quadrupoles was measured using a rotating coil system. The experimental setup and the results obtained are summarized in this section. 


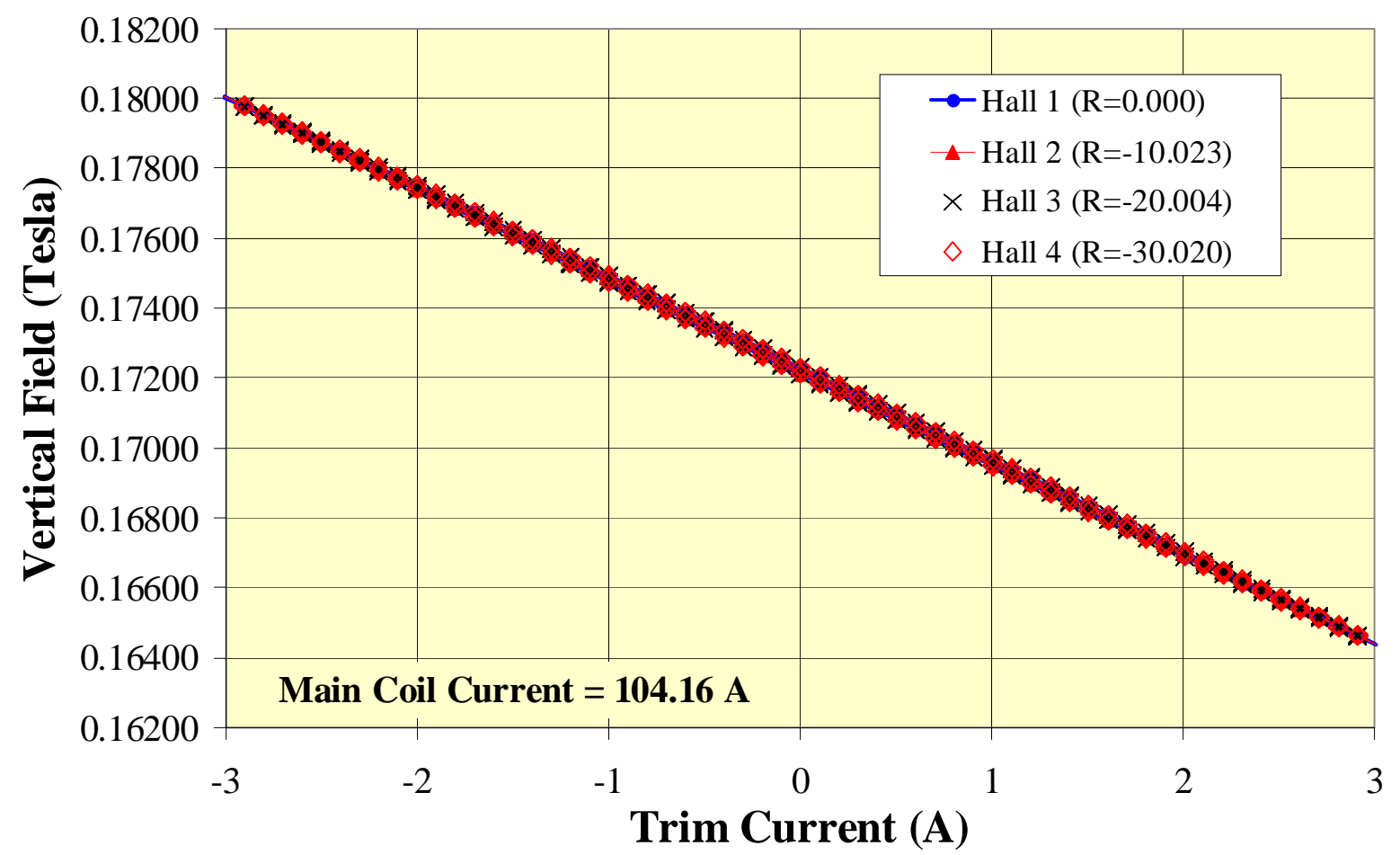

Fig. 9 Excitation curves in the trim coil with the main coil set to $104 \mathrm{~A}(0.172 \mathrm{~T})$ in 3D60 dipole \#5.

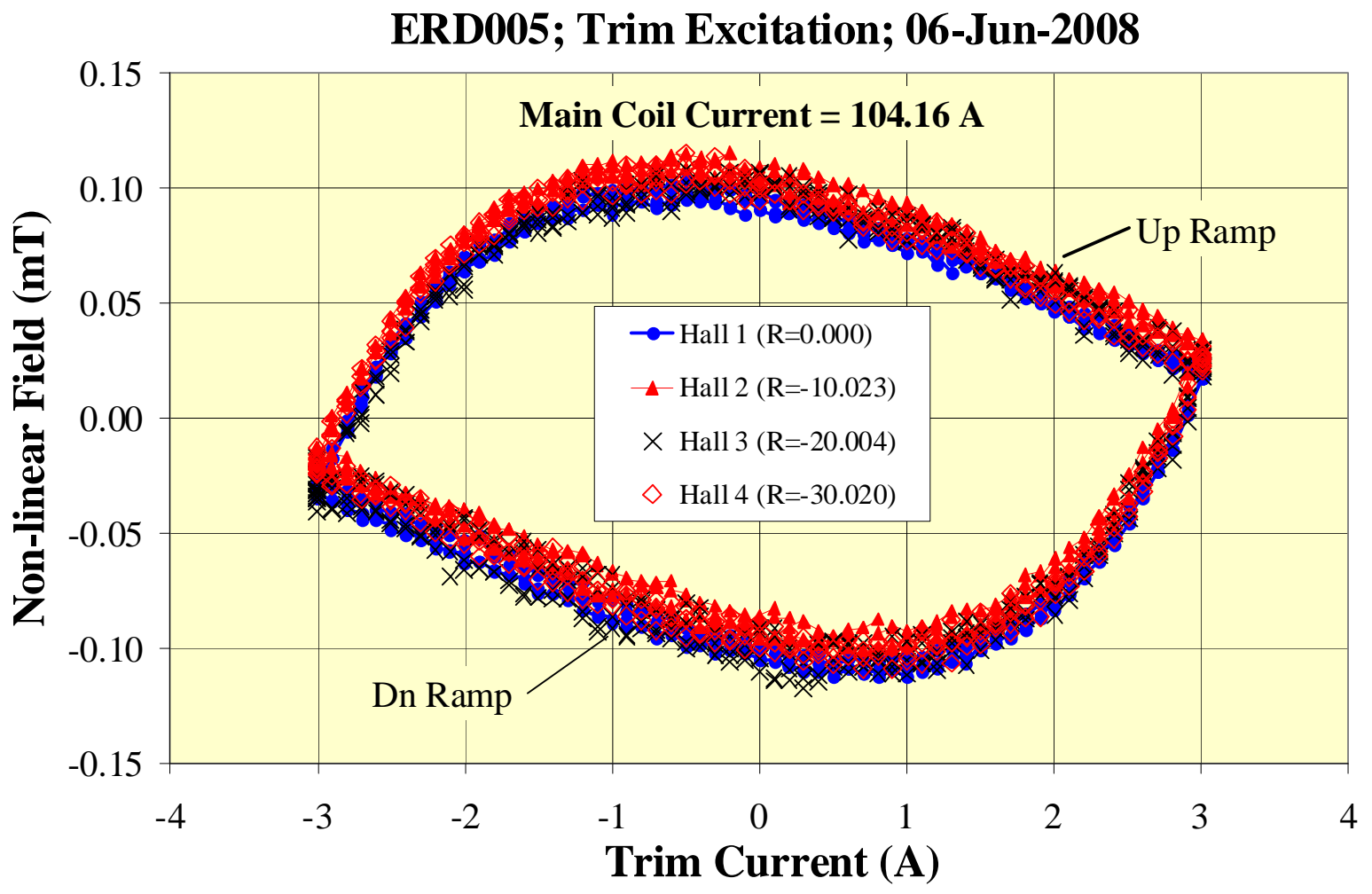

Fig. 10 Non-linear portion of the trim coil field measured in two successive cycles in the current range $\pm 3 \mathrm{~A}$. Cycle to cycle consistency is better than $10 \mu \mathrm{T}$. 


\subsection{Rotating Coil Setup for Magnetic Measurements of 6Q12 Quadrupoles}

The geometry of these quadrupoles is well suited for measurements of the integral field harmonics using rotating coils. The radius of interest in these magnets is $25 \mathrm{~mm}$. An existing rotating coil from a RHIC mole system was retrofitted in a new measurement setup for these measurements. The rotating coil has a set of five tangential windings - a main winding with 15 degrees opening angle and 30 turns, a pair of dipole bucking windings with 3 turns each, and a pair of quadrupole bucking windings with 3 turns each. All windings are at a radius of $27.4 \mathrm{~mm}$, and are 1 meter long. The bucking windings are used to subtract out the large quadrupole as well as any dipole terms in the signal in the main winding in a digital bucking scheme. The bucking makes the measurements relatively insensitive to small imperfections in the rotation of the coil, such as wobble and torsional vibrations.

The rotating coil setup for measuring the 6Q12 quadrupoles is shown in Fig. 11. The coil is rotated using an external drive motor with a period of $3.5 \mathrm{~s}$. An angular encoder is used to generate triggers for data acquisition at 128 evenly distributed angles per revolution. A gravity sensor is mounted to the encoder platform to correlate the measurement frame with gravity. The geometric parameters of the various windings were obtained from a set of detailed calibration runs in a reference dipole (with NMR), a quadrupole, and a sextupole magnet. The calibration procedure was similar to that followed for another rotating coil used for a prototype quadrupole, and is described in detail in [2]. It is estimated that the absolute value of the integrated quadrupole field could be measured to an accuracy of $\pm 0.05 \%$, corresponding to an uncertainty of only $\sim \pm 5 \mu \mathrm{m}$ in the effective radius of the windings.

The magnet is mounted on a level aluminum stand having sockets for a pair of tooling balls at the bottom of the magnet. The ideal geometric axis is defined as a line parallel to the line joining these tooling balls, and offset vertically by a design distance. The axis of the rotating

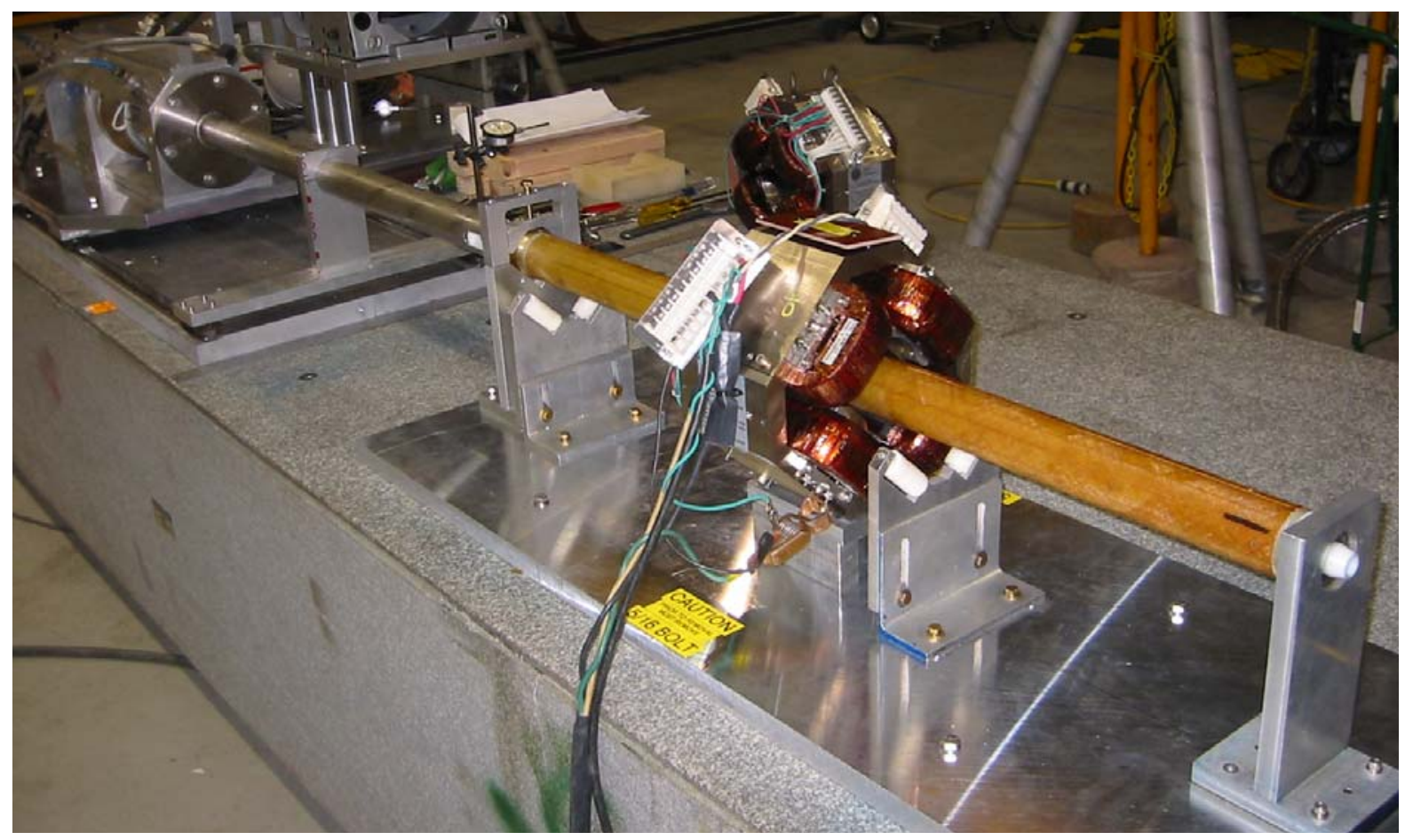

Fig. 11 Rotating coil setup for field quality measurements in 6Q12 quadrupoles. 
coil is precisely aligned to the ideal geometric axis within $\sim 10 \mu \mathrm{m}$. The coil assembly is mounted on precision rails, and can be safely moved in an out of the magnet while supported by a set of rollers. The bearings on the two ends of the coil are held rigidly fixed in relation to the magnet stand, thus maintaining the alignment.

The alignment of the rotating coil axis in the horizontal direction is verified by carrying out measurements both before and after flipping the magnet 180 degrees about a vertical axis. Every magnet was measured twice in this way in order to remove any systematic alignment errors in the horizontal direction, as well as to correct for any systematic errors in the roll angle measurements. This also allowed a check of the alignment stability of the rotating coil. A similar exercise was done for the vertical direction by turning the magnet upside down for the first couple of magnets. However, it was not possible to do this for every magnet during the production due to interference with the terminal mounting blocks. The consistency between the vertical offsets measured in the two horizontally flipped positions of the magnet was better than $\pm 10 \mu \mathrm{m}$. As a result of these detailed measurements, it is estimated that the magnetic center in these quadrupoles was measured to better than $\pm 5 \mu \mathrm{m}$ in the horizontal direction, and to better than $\pm 10 \mu \mathrm{m}$ in the vertical direction. Similarly, the magnetic roll angle with respect to the bottom surface of the magnet was measured to better than $\pm 50 \mu \mathrm{rad}$. This is demonstrated by three different measurements in the same magnet made at three different times during the production phase of these quadrupoles, as shown in Figs. 12 and 13 for the horizontal offset and the roll angle respectively. The data from the two measurements are shown by the blue circles and the red triangles. In the absence of any systematic offset, the two measurements should be equal and opposite. The pink dashed line shows the derived systematic error, and the black line shows the corrected values. As can be seen from these figures, even though the systematic error (pink curve) shows slightly more variation, the final corrected values of the offset and roll angle are consistent within $\pm 1 \mu \mathrm{m}$ and $\pm 15 \mu \mathrm{rad}$ respectively.

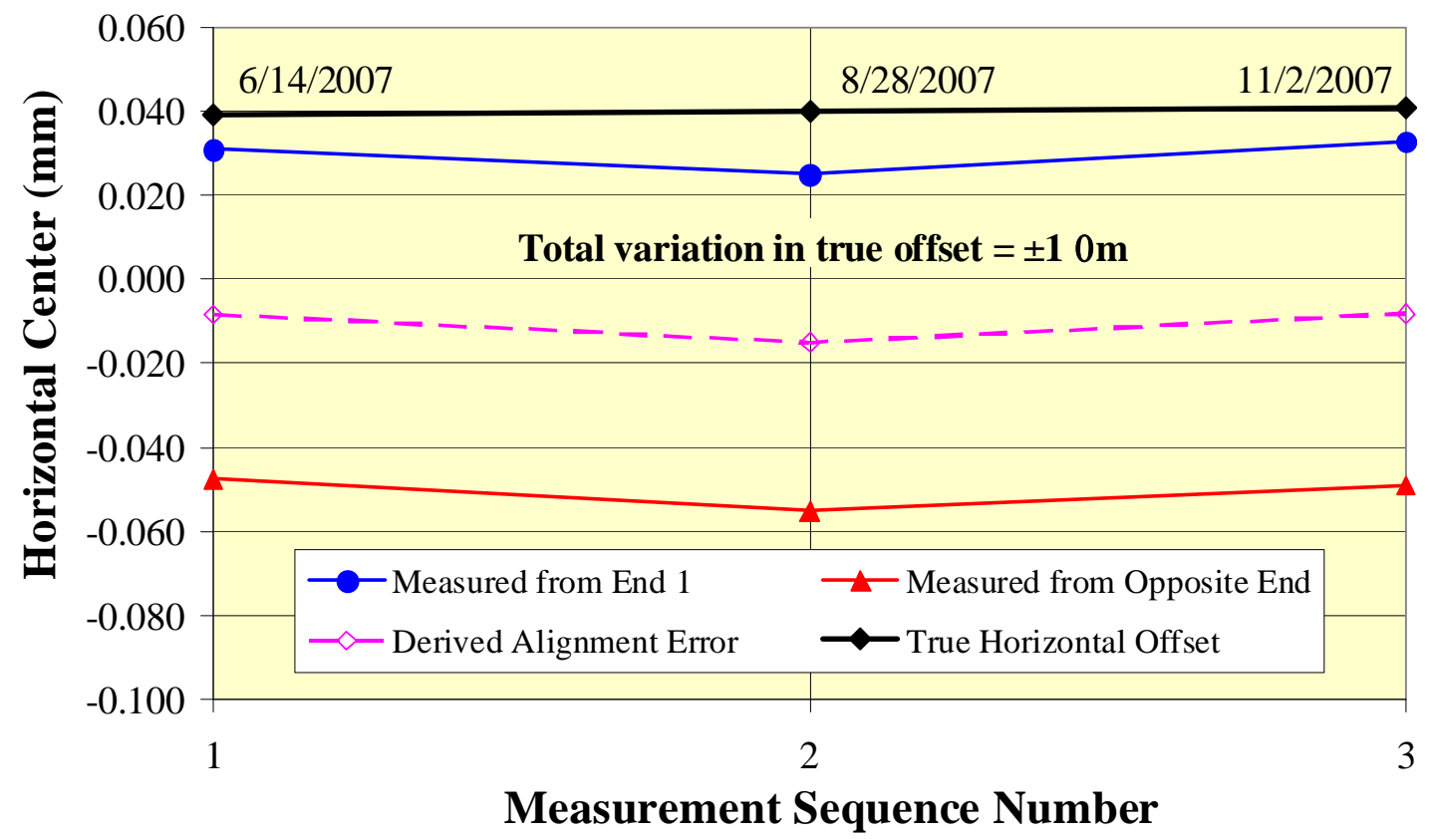

Fig. 12 Reproducibility of horizontal offset measurements in 6Q12 quadrupole \#6. 


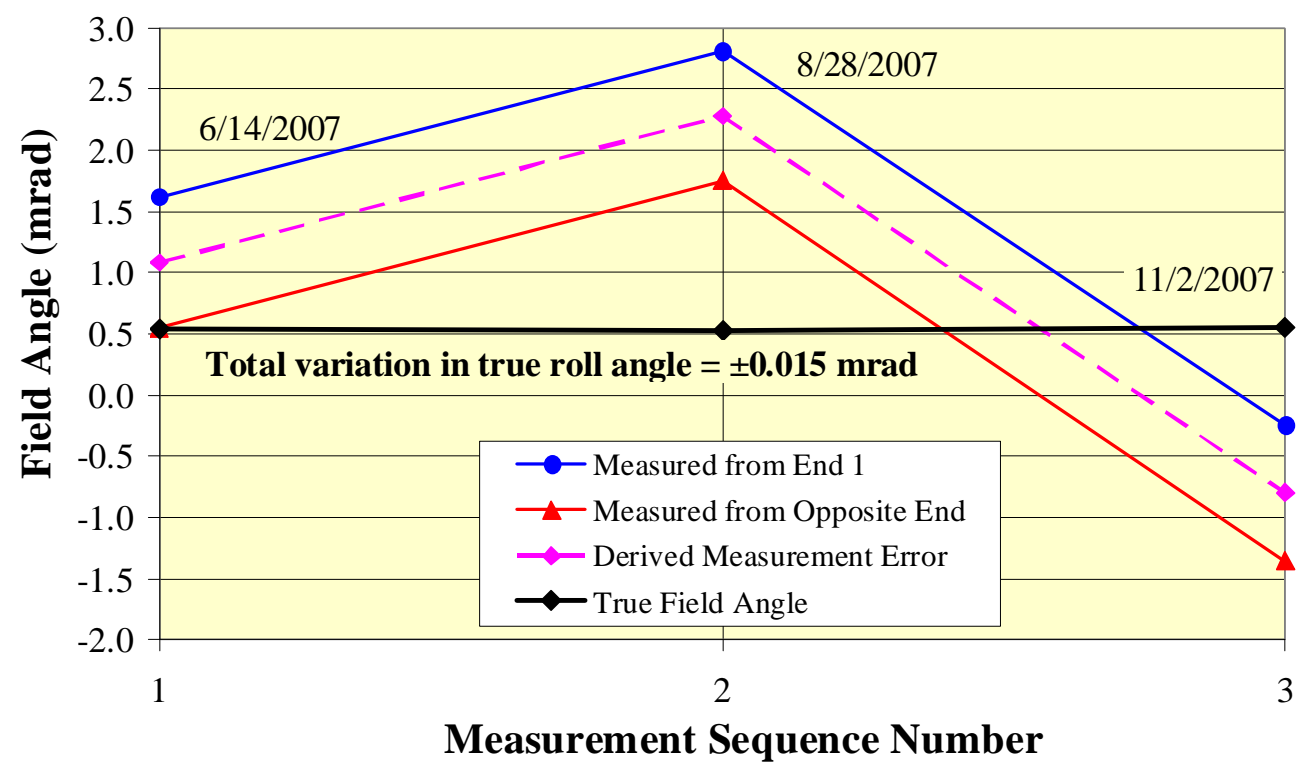

Fig. 13 Reproducibility of horizontal offset measurements in 6Q12 quadrupole \#6.

\subsection{Excitation Curves in the Main Coil}

The excitation curves in the main quadrupole coil were measured for three successive cycles from $0 \mathrm{~A}$ to $6.4 \mathrm{~A}$ and back, after first setting the iron history by carrying out five such cycles without taking data. Fig. 14 shows the measured integral transfer function as a function of current for three cycles. The up and the down ramp difference clearly shows the hysteresis effects, and excellent cycle-to-cycle reproducibility is seen. It is also clear that the magnet does not show any noticeable saturation at the maximum current of $6.4 \mathrm{~A}$. An average integral transfer function is derived from the slope of a linear fit of the quadrupole field to the

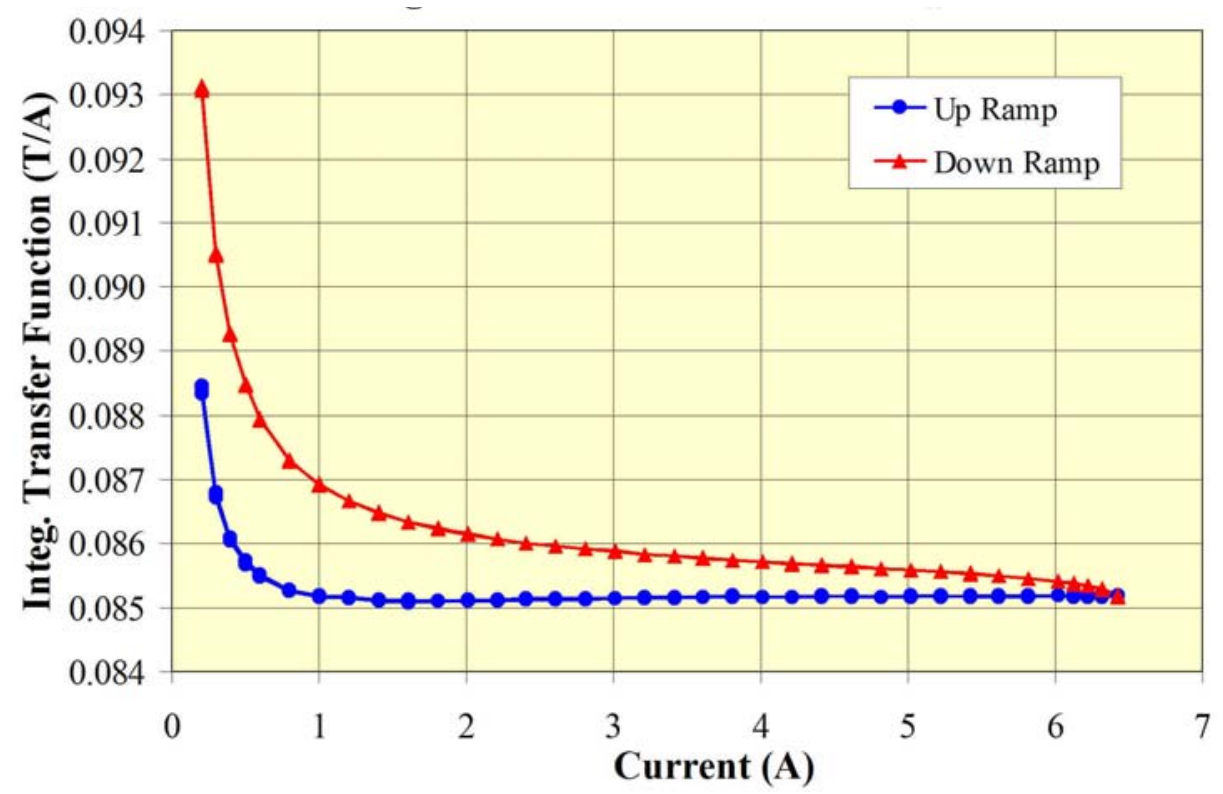

Fig. 14 Integral transfer function $\left(\int G . d \ell / I\right)$ measured in three successive cycles in 6Q12 magnet \# 13. Data from the three cycles are practically indistinguishable. 
current. Fig. 15 shows the average integral transfer function for all the magnets tested. The standard deviation including all magnets is $0.63 \%$. A clear change in the transfer function is seen starting at magnet \#8. This is most likely a result of about $0.2 \mathrm{~mm}$ change in the overall magnet size seen in the mechanical inspection data [3].

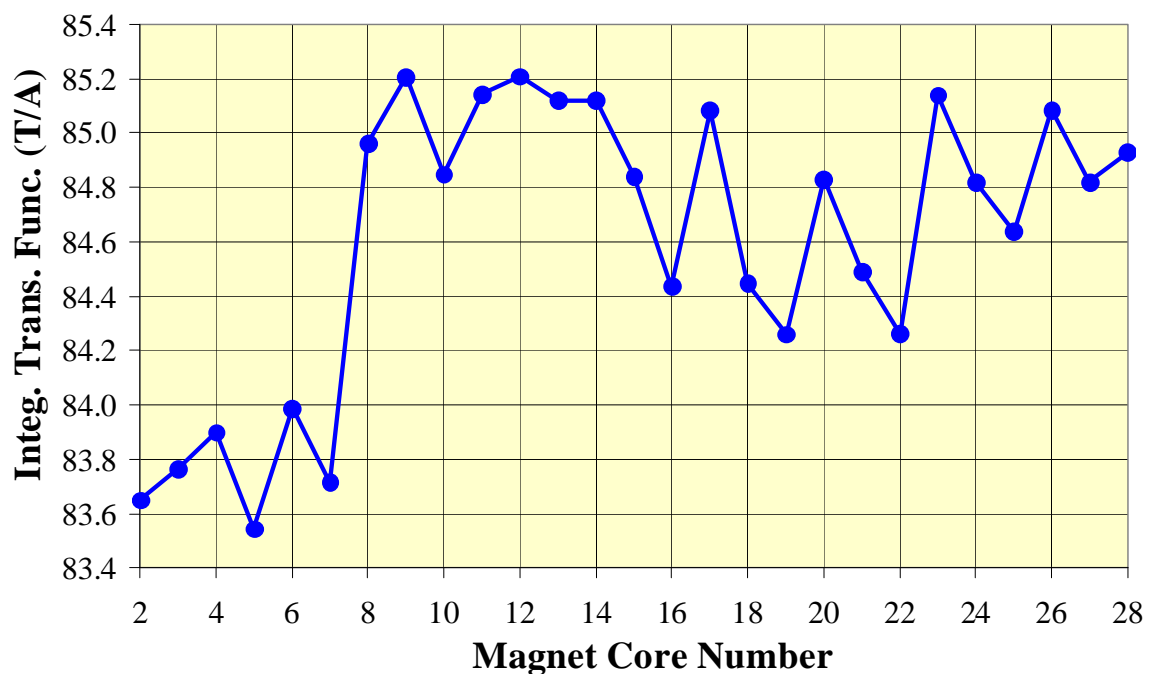

Fig. 15 Integral transfer function in all the 6Q12 quadrupole magnets.

\subsection{Magnetic Center and Roll Angle}

The magnetic center with respect to the "ideal" geometric axis and the magnetic roll angle with respect to the bottom surface of the magnet were obtained from the measured dipole and skew quadrupole components. The rotation axis of the measuring coil coincided with the ideal geometric axis within approximately $10 \mu \mathrm{m}$, as described earlier. The procedure to check and correct for systematic errors was also described earlier in Sec. 3.1. The horizontal and vertical offsets measured in all the magnets are shown in Fig. 16. With only a single exception, the magnetic center in all the magnets coincides with the "ideal" geometric center within $\pm 0.15 \mathrm{~mm}$.

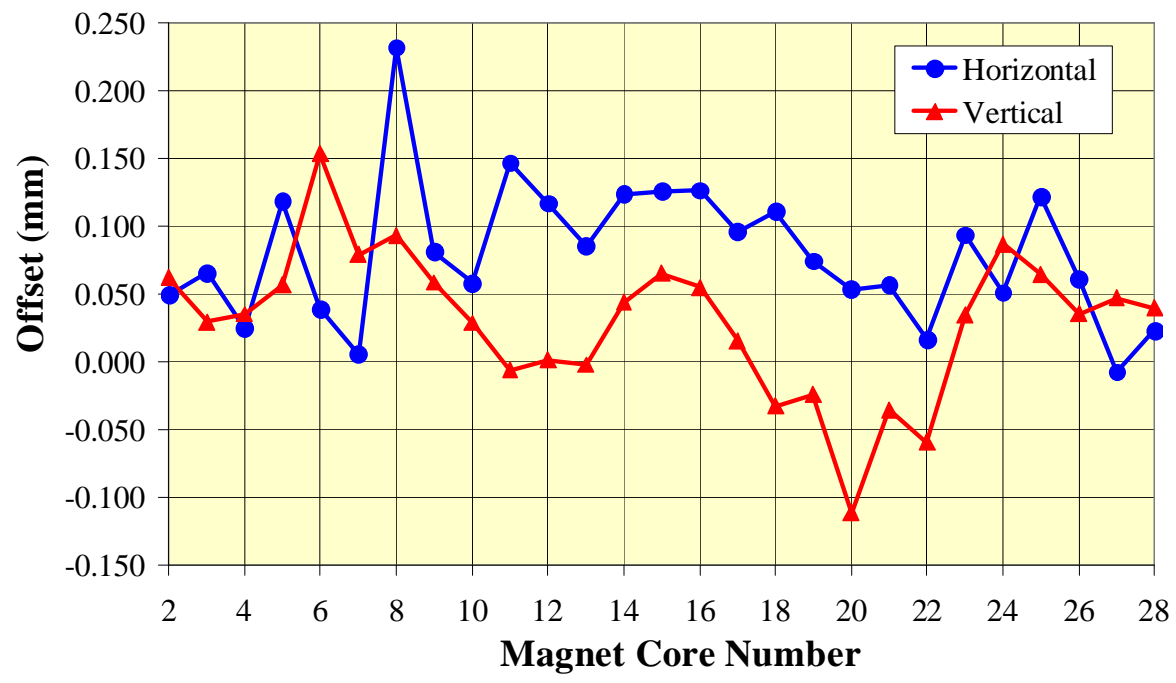

Fig. 16 Magnetic axis relative to the "ideal” geometric axis in the 6Q12 quadrupoles. 
Fig. 17 shows the magnetic roll angle for all the 6Q12 quadrupoles. Most magnets fall within the range $\pm 0.5 \mathrm{mrad}$, with only a few exceptions.

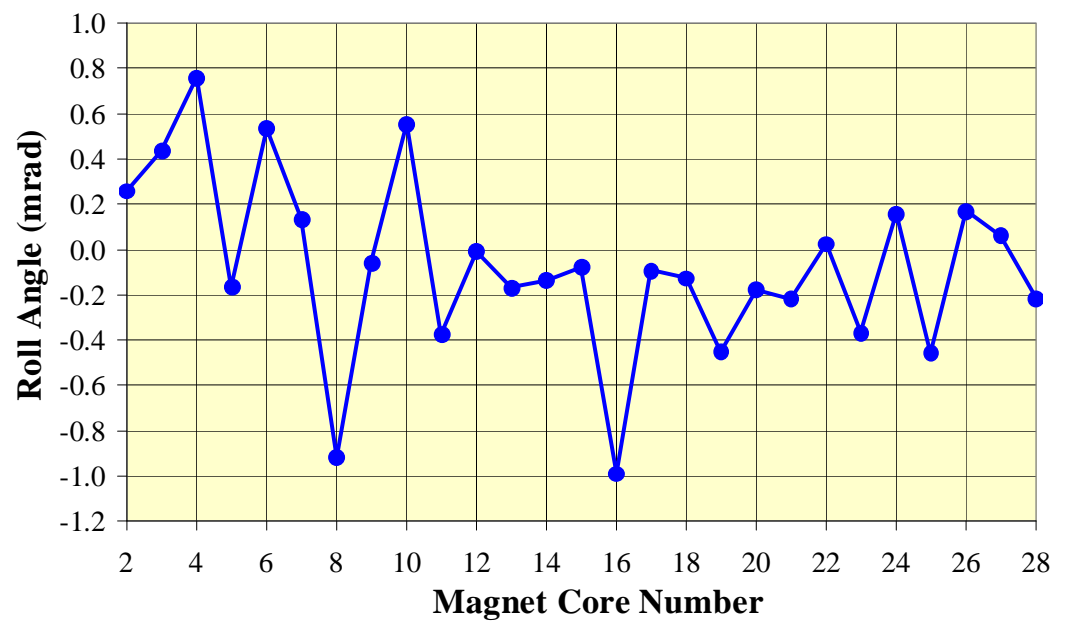

Fig. 17 Magnetic roll angle relative to the bottom surface in the 6Q12 quadrupoles.

\subsection{Field Harmonics in the 6Q12 Quadrupoles}

The Fourier analysis of the rotating coil data also provides all the higher order field harmonics. These harmonics, normalized to the main quadrupole field, were computed at a reference radius of $25 \mathrm{~mm}$, which is the radius of the desired "good field" region. The choice of a rotating coil with radius of $27.4 \mathrm{~mm}$ allows accurate measurements of the higher order harmonics, and terms up to the 30-pole are reported. Excitation curve measurements give a detailed behavior of every harmonic as a function of current, including any non-linearity or hysteresis. In view of the nearly linear nature of these magnets, only the results at $6 \mathrm{~A}$ on the up ramp are summarized here for simplicity. The mean and standard deviations of all harmonics measured in all the 27 6Q12 magnets are given in Table I. The harmonics are expressed in "units" of $10^{-4}$ of the quadrupole field at a radius of $25 \mathrm{~mm}$.

Table I Summary of field harmonics in all 6Q12 quadrupoles at 6 A (up ramp).

\begin{tabular}{|c|c|c|c|c|}
\hline \multirow{2}{*}{ Harmonic } & \multicolumn{2}{|c|}{ Normal Terms } & \multicolumn{2}{c|}{ Skew Terms } \\
\cline { 2 - 5 } & Average & Std. Dev. & Average & Std. Dev. \\
\hline Sextupole & -0.4 & 4.9 & 0.4 & 5.7 \\
\hline Octupole & 2.7 & 2.6 & -0.1 & 0.9 \\
\hline Decapole & 0.0 & 0.8 & 0.2 & 0.8 \\
\hline Dodecapole & 1.0 & 2.2 & -0.3 & 0.7 \\
\hline 14-pole & 0.1 & 0.5 & 0.2 & 0.3 \\
\hline 16-pole & 0.2 & 0.4 & 0.3 & 0.4 \\
\hline 18-pole & 0.6 & 0.5 & 0.3 & 0.5 \\
\hline 20-pole & -32.1 & 0.4 & 0.0 & 0.3 \\
\hline 22-pole & 0.0 & 0.2 & -0.1 & 0.1 \\
\hline 24-pole & 0.0 & 0.2 & -0.1 & 0.1 \\
\hline 26-pole & 0.0 & 0.1 & 0.0 & 0.1 \\
\hline 28-pole & 1.0 & 0.1 & -0.1 & 0.1 \\
\hline 30-pole & 0.0 & 0.1 & 0.0 & 0.0 \\
\hline
\end{tabular}




\subsection{Measurements of Trim Coil Fields}

Each of the four poles is equipped with a trim coil that can carry up to 0.55A. These trim coils can be connected in series to produce either a horizontal steering (normal) or a vertical steering (skew) dipole corrector. The field quality in the trim coils was measured in both configurations. In each configuration, excitation curves were measured between $-0.55 \mathrm{~A}$ to $+0.55 \mathrm{~A}$ in $0.1 \mathrm{~A}$ steps. Three such excitation cycles were measured after first carrying out 5 standardization cycles. The main quadrupole coil was powered at $3.2 \mathrm{~A}$ during these measurements. The trim coils produce roughly $5 \times 10^{-4}$ T.m (500 Gauss.cm) of dipole field at $0.55 \mathrm{~A}$. Since the pole tips and the coil configuration are not optimized to produce a dipole field, the harmonics are rather large. The dominant terms (at $25 \mathrm{~mm}$ radius, in relation to the dipole field produced) are the sextupole (45\%), the decapole (3\%) and the 14-pole (1.8\%). These are the terms that are allowed by the symmetry of the system. The other unallowed terms are well below $1 \%$.

\section{Magnetic Measurements in the Injection Line Dipoles:}

There are two magnets each of the $15^{\circ}$ and $30^{\circ}$ combined function Z-bend magnets in the injection line [1]. The main coils in these magnets have 56 turns per pole, and produce a vertical bending dipole field (skew dipole) of approximately $19 \mathrm{mT}$ at a maximum operating current of $10 \mathrm{~A}$. The core lengths of the $15^{\circ}$ and $30^{\circ}$ magnets are $139.7 \mathrm{~mm}$ and $241.3 \mathrm{~mm}$ respectively, with the corresponding magnetic lengths being $192.2 \mathrm{~mm}$ and $293.9 \mathrm{~mm}$. To achieve the desired bending of $15^{\circ}$ and $30^{\circ}$, the operating currents are $\sim 7.4 \mathrm{~A}$ and $\sim 10 \mathrm{~A}$. In addition to the main dipole coil, these magnets also have horizontal bending corrector dipole (normal dipole), normal quadrupole, and skew sextupole coils, each capable of operating at a maximum current of $\pm 0.2 \mathrm{~A}$.

The injection line dipoles have an aperture of approximately $6 \mathrm{~cm}$ by $9 \mathrm{~cm}$. It is therefore convenient to measure the field quality in these magnets using a rotating coil system. The rotating coil system described in Sec. 3.1 for measurements of the 6Q12 quadrupole was used for measuring these injection line magnets also. The results of these measurements provide the integrated main fields produced by each of the coils, as well as the field harmonics at a reference radius of $25 \mathrm{~mm}$. For the purposes of magnetic measurements data recording, the two $15^{\circ}$ magnets were named ERL151 and ERL152, and the two $30^{\circ}$ magnets were named ERL301 and ERL302.

\subsection{Measurements of the Main Dipole Coil Fields}

The integrated field harmonics of the main coil were measured in a complete excitation cycle from $0 \mathrm{~A}$ to $10 \mathrm{~A}$ and back in $0.5 \mathrm{~A}$ steps (21 current steps). Prior to these measurements, the magnet was cycled 5 times between $0 \mathrm{~A}$ and $10 \mathrm{~A}$ in order to set the magnet iron history. Two complete excitation cycles were measured back-to-back to look for cycle to cycle consistency. No appreciable difference was seen between the two cycles. All other correction coils were left unpowered during these measurements.

An example of the integrated dipole and the sextupole terms as a function of current is shown in Figs. 18 and 19 for a $15^{\circ}$ dipole, ERL152. The slopes of straight line fits to these data provide the geometric values of the dipole and sextupole harmonics. The geometric values of 

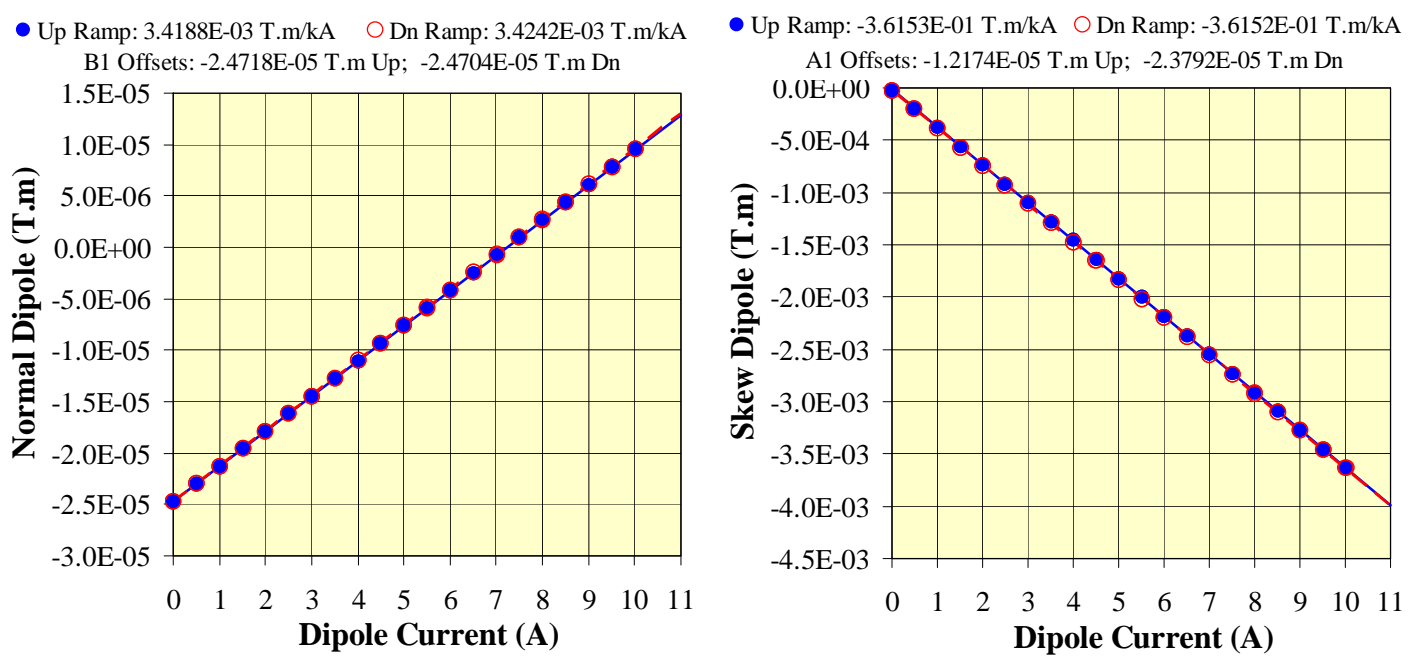

Fig. 18 Normal (B1) and skew (A1) dipole terms from the main coil as a function of current in the $15^{\circ}$ injection line magnet ERL152. The B1 term is a result of angular misalignment $\left(\sim 0.5^{\circ}\right)$ between the dipole field and the measuring coil reference frame.

all other harmonics are similarly determined. It can be seen that the sextupole term (at a reference radius of $25 \mathrm{~mm}$ ) in this magnet is $\sim 0.5 \%$ of the main dipole term. The next allowed term, decapole, is $\sim 0.15 \%$ and the unallowed quadrupole term is $\sim 0.22 \%$. All other terms are well below $0.1 \%$. The averages of up and down ramp values of all the harmonics in all the four injection line dipoles are summarized in Table II. The harmonics are evaluated at reference radius of $25 \mathrm{~mm}$ and are expressed as percentage of the main dipole field. The values presented in the table are the magnitudes, $\left|B n+i A_{n}\right|$, of the $2 n$-pole harmonic, where $B_{n}$ and $A_{n}$ are the normal and the skew components respectively. Harmonics above the 22-pole are practically zero.
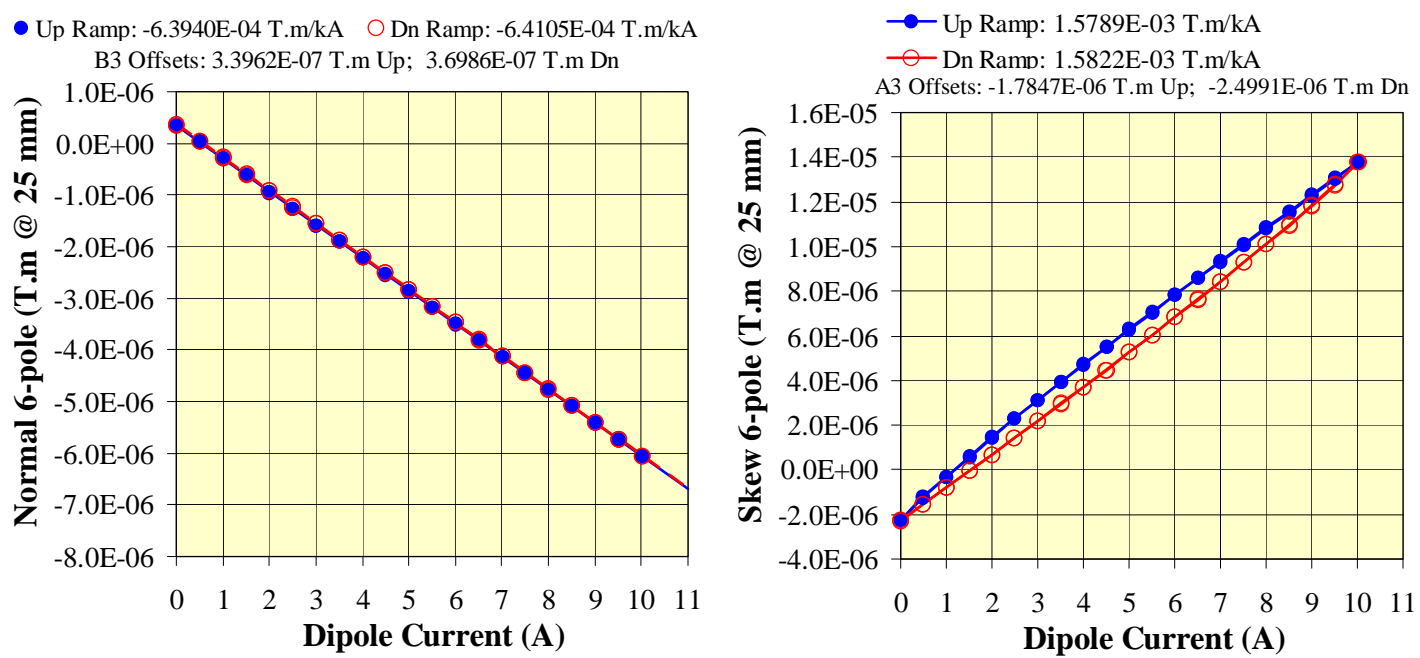

Fig. 19 Normal (B3) and skew (A3) sextupole terms at a reference radius of $25 \mathrm{~mm}$ from the main coil as a function of current in the $15^{\circ}$ injection line magnet ERL152. 
Table II Summary of field harmonics in the main dipole coil in all the four injection line magnets. The reference radius is $25 \mathrm{~mm}$.

\begin{tabular}{|c|c|c||c|c|}
\hline Quantity & ERL151 & ERL152 & ERL301 & ERL302 \\
\hline I.T.F. (T.m/A) & $3.619 \mathrm{E}-04$ & $3.615 \mathrm{E}-04$ & $5.522 \mathrm{E}-04$ & $5.530 \mathrm{E}-04$ \\
\hline Dipole (\%) & 100.00 & 100.00 & 100.00 & 100.00 \\
\hline Quadrupole (\%) & 0.14 & 0.22 & 0.08 & 0.18 \\
\hline Sextupole (\%) & 0.28 & 0.47 & 0.76 & 0.66 \\
\hline Octupole (\%) & 0.05 & 0.03 & 0.04 & 0.06 \\
\hline Decapole (\%) & 0.09 & 0.15 & 0.20 & 0.18 \\
\hline 12-pole (\%) & 0.02 & 0.00 & 0.03 & 0.02 \\
\hline 14-pole (\%) & 0.03 & 0.03 & 0.04 & 0.02 \\
\hline 16-pole (\%) & 0.01 & 0.00 & 0.02 & 0.00 \\
\hline 18-pole (\%) & 0.02 & 0.02 & 0.02 & 0.02 \\
\hline 20-pole (\%) & 0.00 & 0.00 & 0.00 & 0.00 \\
\hline 22-pole (\%) & 0.00 & 0.00 & 0.00 & 0.00 \\
\hline
\end{tabular}

\subsection{Measurements of the Quadrupole Coil Fields}

The excitation curves for the quadrupole coil were measured over the current range of $\pm 0.2 \mathrm{~A}$ in steps of $0.1 \mathrm{~A}$ on both the up and the down ramps. Since the field produced by the quadrupole coil could be affected by the current in the main dipole coil, excitation curves were measured both without any background dipole field, as well as with the main dipole coil set to $5 \mathrm{~A}$ and $10 \mathrm{~A}$. In each case, the slope of a straight line fit of the harmonics as a function of quadrupole coil current give the geometric value of harmonics from the quadrupole coil. An example of the integrated quadrupole field as a function of current is shown in Fig. 20 for
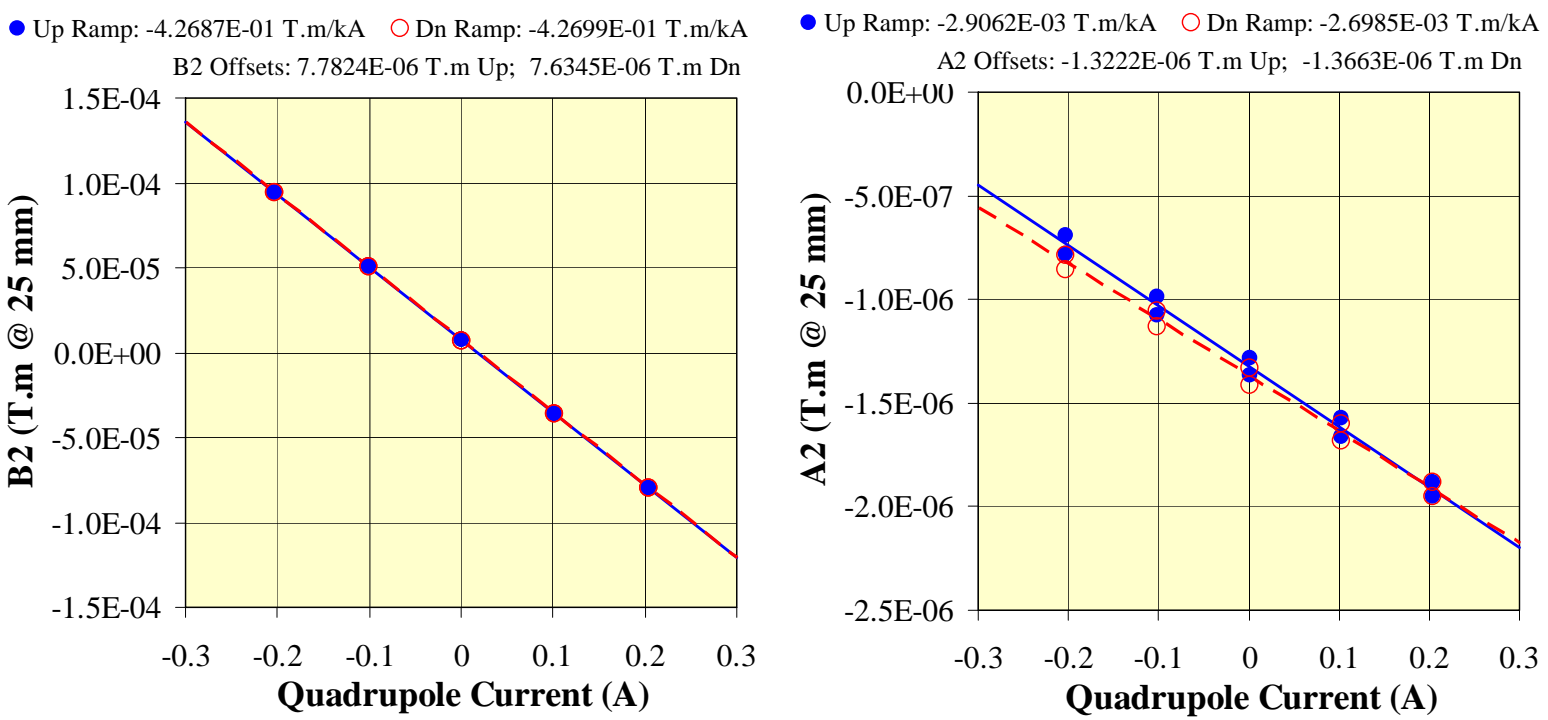

Fig. 20 Normal (B2) and skew (A2) integrated quadrupole terms at a reference radius of $25 \mathrm{~mm}$ as a function of quadrupole current in the $30^{\circ}$ injection line magnet ERL301, with a background field from the main dipole coil powered at $10 \mathrm{~A}$. 
the case where the main dipole coil is set to $10 \mathrm{~A}$. A small current dependence of the skew quadrupole term is a result of $\sim 0.2$ degree misalignment between the quadrupole field and the reference frame of the measurement system. The averages of up and down ramp values of all the harmonics in the quadrupole coils of all the four injection line magnets are summarized in Table III. The values are normalized to the strength of the quadrupole field in this case. The

Table III Summary of field harmonics in the quadrupole coil in all the four injection line magnets with the main dipole at $10 \mathrm{~A}$. The reference radius is $25 \mathrm{~mm}$.

\begin{tabular}{|c|c|c||c|c|}
\hline Quantity & ERL151 & ERL152 & ERL301 & ERL302 \\
\hline I.T.F.(T/A) & $1.088 \mathrm{E}-02$ & $1.094 \mathrm{E}-02$ & $1.708 \mathrm{E}-02$ & $1.717 \mathrm{E}-02$ \\
\hline Dipole (\%) & 1.47 & 4.75 & 3.40 & 1.63 \\
\hline Quadrupole (\%) & 100.00 & 100.00 & 100.00 & 100.00 \\
\hline Sextupole (\%) & 0.19 & 0.70 & 0.61 & 0.62 \\
\hline Octupole (\%) & 1.92 & 2.30 & 2.05 & 2.04 \\
\hline Decapole (\%) & 0.12 & 0.12 & 0.02 & 0.05 \\
\hline 12-pole (\%) & 0.09 & 0.07 & 0.06 & 0.01 \\
\hline 14-pole (\%) & 0.05 & 0.07 & 0.01 & 0.02 \\
\hline 16-pole (\%) & 0.12 & 0.11 & 0.11 & 0.14 \\
\hline 18-pole (\%) & 0.01 & 0.01 & 0.01 & 0.01 \\
\hline 20-pole (\%) & 0.02 & 0.02 & 0.02 & 0.02 \\
\hline 22-pole (\%) & 0.00 & 0.00 & 0.01 & 0.00 \\
\hline
\end{tabular}

integral transfer function, as well as the harmonics, were found to be nearly the same for all the three different excitations of the main dipole coil.

\subsection{Measurements of the Horizontal (Normal) Corrector Dipole Coil Fields}

The measurements of fields from for the horizontal dipole corrector (normal dipole) coil were carried out over the current range of $\pm 0.2 \mathrm{~A}$, as in the case of the quadrupole coil described above. In this case, the measurements were made with the main dipole coil powered at either
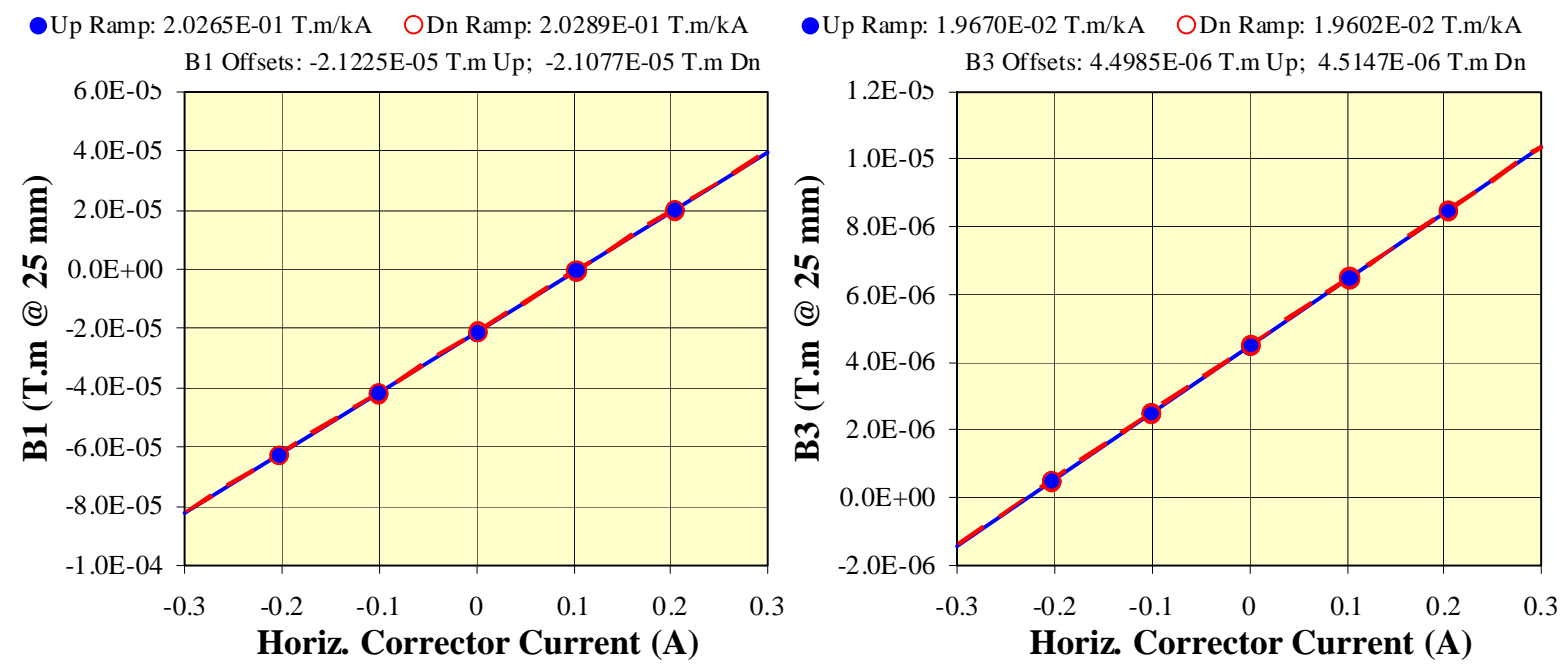

Fig. 21 Integrated normal dipole (B1) and normal sextupole (B3) terms as a function of horizontal corrector current in the $15^{\circ}$ injection line magnet ERL151, with a background field from the main dipole coil powered at $10 \mathrm{~A}$. 
$5 \mathrm{~A}$ or $10 \mathrm{~A}$. No measurements were made with the main dipole coil turned off. Fig. 21 shows the current dependence of the normal dipole and the normal sextupole terms in the magnet ERL151, with the main dipole coil powered at $10 \mathrm{~A}$. The sextupole term from the

Table IV Summary of field harmonics in the horizontal corrector coil in all the four injection line magnets with the main dipole at $10 \mathrm{~A}$. The reference radius is $25 \mathrm{~mm}$.

\begin{tabular}{|c|c|c||c|c|}
\hline Quantity & ERL151 & ERL152 & ERL301 & ERL302 \\
\hline I.T.F. (T.m/A) & $2.028 \mathrm{E}-04$ & $2.045 \mathrm{E}-04$ & $2.979 \mathrm{E}-04$ & $3.015 \mathrm{E}-04$ \\
\hline Dipole (\%) & 100.00 & 100.00 & 100.00 & 100.00 \\
\hline Quadrupole (\%) & 0.14 & 1.19 & 0.60 & 0.66 \\
\hline Sextupole (\%) & 9.68 & 10.05 & 10.55 & 10.64 \\
\hline Octupole (\%) & 0.17 & 0.17 & 0.04 & 0.13 \\
\hline Decapole (\%) & 0.20 & 0.03 & 0.11 & 0.15 \\
\hline 12-pole (\%) & 0.06 & 0.06 & 0.01 & 0.01 \\
\hline 14-pole (\%) & 0.16 & 0.12 & 0.14 & 0.16 \\
\hline 16-pole (\%) & 0.01 & 0.02 & 0.01 & 0.01 \\
\hline 18-pole (\%) & 0.00 & 0.02 & 0.01 & 0.01 \\
\hline 20-pole (\%) & 0.01 & 0.00 & 0.01 & 0.00 \\
\hline 22-pole (\%) & 0.00 & 0.00 & 0.00 & 0.01 \\
\hline
\end{tabular}

horizontal corrector is $\sim 10 \%$ of the dipole field produced by it. The results in all the four injection line magnets are summarized in Table IV.

\subsection{Measurements of the Skew Sextupole Corrector Coil Fields}

The measurements of fields from for the skew sextupole corrector coil were carried out over the current range of $\pm 0.2 \mathrm{~A}$, as in the case of the other corrector coils described above. In this case, the measurements were made with the main dipole coil powered at either $5 \mathrm{~A}$ or $10 \mathrm{~A}$. No measurements were made with the main dipole coil turned off. Fig. 22 shows the current
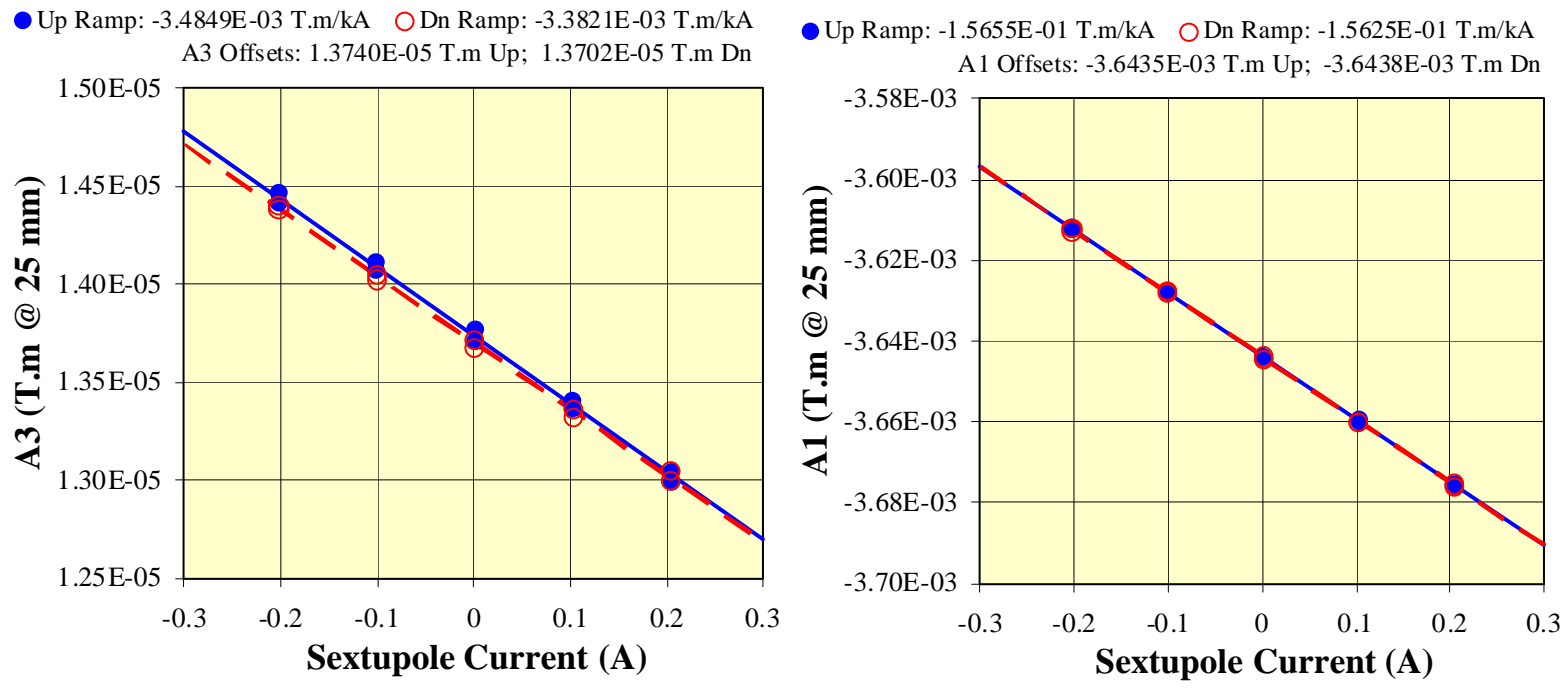

Fig. 22 Integrated skew sextupole (A3) and skew dipole (A1) terms as a function of horizontal corrector current in the $15^{\circ}$ injection line magnet ERL152, with a background field from the main dipole coil powered at $10 \mathrm{~A}$. 
dependence of the skew sextupole and the skew dipole terms in the magnet ERL152, with the main dipole coil powered at $10 \mathrm{~A}$. It should be noted that the sextupole coil produces also a strong skew dipole term which is $\sim 46$ times stronger than the sextupole field produced by it. To put this in the right perspective, the sextupole corrector operating at the maximum $0.2 \mathrm{~A}$ produces as much skew dipole as the main coil can produce at about $0.1 \mathrm{~A}$. This shows that the settings for the main dipole coils may have to be adjusted slightly depending upon the settings of the sextupole coils. The results for skew sextupole coils in all the four injection line magnets are summarized in Table $\mathrm{V}$ for the case where the maind dipole coil is powered

Table V Summary of field harmonics in the sextupole coil in all the four injection line magnets with the main dipole at $10 \mathrm{~A}$. The reference radius is $25 \mathrm{~mm}$.

\begin{tabular}{|c|c|c||c|c|}
\hline Quantity & ERL151 & ERL152 & ERL301 & ERL302 \\
\hline I.T.F.* (T/m/A) & $5.544 \mathrm{E}-03$ & $5.494 \mathrm{E}-03$ & $8.972 \mathrm{E}-03$ & $9.229 \mathrm{E}-03$ \\
\hline Dipole (\%) & 4610.0 & 4556.1 & 4289.2 & 4160.5 \\
\hline Quadrupole (\%) & 8.07 & 22.46 & 3.06 & 4.14 \\
\hline Sextupole (\%) & 100.00 & 100.00 & 99.98 & 100.00 \\
\hline Octupole (\%) & 3.71 & 1.41 & 1.85 & 2.83 \\
\hline Decapole (\%) & 35.61 & 34.81 & 36.75 & 36.86 \\
\hline 12-pole (\%) & 0.65 & 0.03 & 0.21 & 0.59 \\
\hline 14-pole (\%) & 4.78 & 4.78 & 4.80 & 4.88 \\
\hline 16-pole (\%) & 0.10 & 0.04 & 0.13 & 0.02 \\
\hline 18-pole (\%) & 0.24 & 0.28 & 0.10 & 0.21 \\
\hline 20-pole (\%) & 0.01 & 0.02 & 0.15 & 0.01 \\
\hline 22-pole (\%) & 0.01 & 0.03 & 0.22 & 0.02 \\
\hline
\end{tabular}

$* \int B^{\prime \prime} . d \ell=2 \times$ I.T.F. $\times$ Current for sextupole

to $10 \mathrm{~A}$. The sextupole transfer functions measured with the main dipole coil powered at $5 \mathrm{~A}$ are within $\sim 1 \%$ of the values in Table $\mathrm{V}$.

\section{References:}

[1] G. Mahler, ERL Magnet Systems, AP Report C-A/AP/375, January 2010.

[2] A. Jain and R. Thomas, Fiducialization and Calibration of Rotating Coil for Magnetic Measurements of the ERL Quadrupoles, Magnet Division Note 646-11 (AM-MD-346), December 13, 2006. (Available on the web at: http://www.bnl.gov/magnets/magnet_files/Publications/MDN-646-11.pdf.)

[3] S. Seberg, Private Communication. 